\title{
Canadian Urological Association best practice report: Prostate-specific membrane antigen positron emission tomography/computed tomography (PSMA PET/CT) and PET/magnetic resonance $(\mathrm{MR})$ in prostate cancer
}

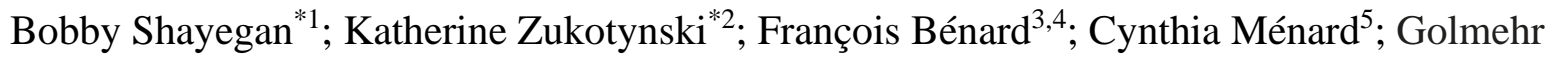
Sistani $^{6}$; Glenn Bauman ${ }^{7}$; Patrick Veit-Haibach ${ }^{8}$; Ur Metser ${ }^{9}$

${ }^{1}$ Department of Urology, McMaster University, Hamilton, ON, Canada; ${ }^{2}$ Departments of Medicine and Radiology, McMaster University, Hamilton, ON, Canada; ${ }^{3}$ PET Functional Imaging, BC Cancer, Vancouver, BC, Canada; ${ }^{4}$ Department of Radiology, University of British Columbia, Vancouver, BC, Canada; ${ }^{5}$ Department of Radiation Oncology, Université de Montréal, Montreal, QC, Canada; ${ }^{6}$ Department of Medical Imaging, Western University, London, ON, Canada; ${ }^{7}$ Department of Oncology, Western University, London, ON, Canada;

${ }^{8}$ Joint Department of Medical Imaging, University of Toronto, Toronto, ON, Canada

*Equal contributors

Cite as: Shayegan B, Zukotynski K, Bénard F, et al. Canadian Urological Association best practice report: Prostate-specific membrane antigen positron emission tomography/computed tomography (PSMA PET/CT) and PET/magnetic resonance (MR) in prostate cancer. Can Urol Assoc J 2021 March 1; Epub ahead of print. http://dx.doi.org/10.5489/cuaj.7268

Published online March 1, 2021

$* * *$

\begin{abstract}
Prostate-specific membrane antigen (PSMA)-targeted positron emission tomography (PET) is increasingly being used worldwide as part of the clinical workup for men with prostate cancer. With high overall accuracy for the detection of prostate cancer, PSMA-targeted PET has an increasingly established role in the setting of biochemical failure after primary therapy and an evolving role in the setting of initial disease staging; its utility for guiding management in the setting of metastatic disease is less clear. Although the specificity is high, familiarization with potential pitfalls in the interpretation of PSMA-targeted PET, including knowledge of the causes for false positive and negative examinations, is critical. The aim of this article is to provide an illustrative discussion of the current and evolving clinical indications for PSMA-targeted PET, as well as a review of physiologic radiopharmaceutical biodistribution and potential imaging pitfalls.
\end{abstract}




\section{Introduction}

Prostate-specific membrane antigen (PSMA), also known as glutamate carboxypeptidase II (GCP II), is a transmembrane glycoprotein highly expressed in prostate cancer cells [1, 2]. The glycoprotein acts as a glutamate carboxypeptidase on various substrates and its enzymatic activity allows for the development of specific inhibitors that are internalized after ligand binding. PSMA expression tends to increase with increased pathological Gleason grade and is thought to be upregulated with the emergence of androgen independence [3, 4]. Both Gallium-68 $\left({ }^{68} \mathrm{Ga}\right)$ and Fluorine-18 $\left({ }^{18} \mathrm{~F}\right)$ labeled PSMA-specific radiopharmaceuticals are clinically used. ${ }^{68} \mathrm{Ga}$ has a half-life of 68 minutes and is typically derived from a generator, while ${ }^{18} \mathrm{~F}$ has a halflife of 110 minutes and is produced using a cyclotron. The longer half-life of ${ }^{18} \mathrm{~F}$ facilitates offsite production and shipping of ${ }^{18} \mathrm{~F}$ labeled PSMA-targeted radiopharmaceuticals. Further, these radiopharmaceuticals are thought to be associated with higher image resolution $[5,6]$. Today, the most commonly used PSMA-targeted PET radiopharmaceuticals in Canada are ${ }^{68} \mathrm{Ga}-\mathrm{PSMA}-11$, ${ }^{18} \mathrm{~F}-\mathrm{DCFPyL}$ and ${ }^{18} \mathrm{~F}-\mathrm{PSMA}-1007$. The use of either hybrid PET/CT or PET/MR scanners with PSMA-targeted radiopharmaceuticals allows anatomical localization and characterization of PSMA-avid lesions.

In this article, we: 1. Review image acquisition and normal biodistribution of PSMAtargeted radiopharmaceuticals, including common physiological or non-prostate cancer entities, which may show increased radiopharmaceutical uptake; 2 . Discuss the role of PSMA-targeted $\mathrm{PET} / \mathrm{CT}$ and PET/MR for common prostate cancer indications, including illustrative cases; 3 . Make recommendations for the incorporation of PSMA PET into care paths in the Canadian context. Although we discuss the most common PSMA-targeted radiopharmaceuticals in general, our Figures will focus on ${ }^{18} \mathrm{~F}-\mathrm{DCFPyL}$, currently the most commonly used PSMA-targeted radiopharmaceutical in Canada.

\section{Integrated whole-body PET/CT and PET/MRI protocol}

The acquisition protocol for PSMA-targeted PET/CT and PET/MR is similar. The suggested injected dose and uptake time for the commonly used PSMA-targeted PET radiopharmaceuticals are given in Table 1 [7-9]. After the tracer-specific uptake time, the patient is positioned supine on the imaging table, optimally with arms up. Routine image coverage is from the top of the skull to the mid thighs. Usually, 5-9 bed positions are obtained, depending on the patient height (2-3 $\mathrm{min} / \mathrm{bed}$ position). The method of attenuation correction is dependent on the imaging platform used. For PET/CT, attenuation correction maps are generated from CT data. For PET/MR, the most prevalent attenuation correction methods are MRI-based attenuation correction maps generated from a 2-point Dixon Gradient-echo sequence for the entire scan field [10]. The administration of IV Lasix (10-40 mg) prior to imaging may be helpful to diminish bladder activity (which can obscure uptake in the prostate gland or bed) and ureteral activity (a potential mimic of adenopathy) for agents with primary urinary clearance such as ${ }^{18} \mathrm{~F}-\mathrm{DCFPyL}$. 
A further purpose of the low-dose CT or MR sequences obtained with PET/CT or PET/MR, respectively, is to enable accurate localization of lesions detected on PSMA-targeted PET. To enable localization of small metastases, axial gadolinium-enhanced T1 sequence such as the volumetric interpolated breath-hold examination $[=\mathrm{VIBE}]$ sequence, has shown high lesion conspicuity and is time efficient with overall acquisition time of approximately 6 minutes for a whole body acquisition [11]. Time permitting other sequences may be added, if required, including Coronal T2-weighted turbo inversion recovery magnitude for bone lesions, or diffusion-weighted imaging. Due to time constraints, the use of additional sequences may be limited to cases where there is a clinical need to further characterize lesions identified on PET or on other MR sequences. Dedicated MR imaging of the prostate gland or prostate bed may be needed in specific clinical situations; for example, during the primary staging of high-risk prostate cancer with PET/MR or to confirm local recurrence of disease when PET images are obscured by activity from radiotracer in the bladder. In these instances, routine MR protocols are used. These generally include multiplanar T2-weighted sequences, diffusion-weighted sequences as well as pre and post-dynamic contrast-enhanced MR. The approach of more comprehensive prostate or body imaging typically requires real-time monitoring of the imaging study by a radiologist and/or nuclear medicine physician.

\section{Normal biodistribution of PSMA-targeted radiopharmaceuticals and common PSMA-avid benign entities}

Physiological, high PSMA-targeted radiopharmaceutical uptake is seen in PSMA-expressing organs such as the lacrimal and salivary glands as well as the kidneys. Moderate

radiopharmaceutical uptake is seen in the spleen and nasopharynx, and variable uptake is seen in the vocal cords, trachea and bronchi as well as the proximal gastrointestinal tract (Figure 1A, B) [12]. The degree of uptake in the liver and accumulation in the urinary tract and bladder varies depending on the clearance mechanism of the specific radiopharmaceutical. Most PSMAtargeted radiopharmaceuticals are cleared via the genitourinary tract with high accumulation in the urinary tract and bladder and moderate uptake in the liver [12]. ${ }^{18}$ F-PSMA-1007 is an exception, with primarily hepatobiliary clearance, higher uptake in the liver and little accumulation in the ureters and bladder [13]. Lack of renal clearance may aid in identifying disease sites adjacent to the bladder and ureters, such as local tumor recurrence after prostatectomy. Peripheral neural structures, including the ganglia and nerve roots may show physiologic increased radiopharmaceutical uptake. The celiac and stellate ganglia have been described as potential pitfalls for the assessment of retroperitoneal and supraclavicular lymph node disease spread, respectively (Figure 2).

Despite its name, PSMA is not prostate specific and there are numerous non-prostate malignancies as well as benign processes which have been reported to be PSMA-avid [14]. This may be, in part, related to the expression of PSMA on the cell membrane or increased endothelial PSMA expression in neovasculature. Although the list of benign entities associated with 
increased PSMA uptake is evolving (Table 2), it includes infectious and inflammatory processes (e.g., sarcoidosis) as well as benign neoplasms consisting of mesenchymal tumors (vascular, neurogenic, connective tissue origin), and epithelial tumors including those of thymic origin, and adenomas in various organs. Bone remodeling may also be PSMA-avid, and increased tracer uptake may be seen with healing fractures, as well as degenerative or arthritic processes (e.g., osteophytes), among others. Although not well-understood, increased blood flow and vascular permeability may explain, at least in part, the increased PSMA uptake in inflammatory conditions. Disruption of the blood-brain barrier has been suggested as the etiology for focal abnormal radiopharmaceutical accumulation in acute or subacute ischemic cerebrovascular events [15]. The pattern of distribution and correlation with imaging features may aid in accurately characterizing the benign process (Figure 3 ).

\section{Other (non-prostate) PSMA-avid malignancies}

Several malignancies are PSMA-avid and can have a range of PSMA intensity. As an illustration, Figure 4 shows an intensely PSMA-avid adrenal leiomyosarcoma, while Figure 5 shows intensely PSMA-avid primary thyroid cancer. Uptake in non-small cell lung cancer may be mild or intense (Figure 6). In certain cases, the use of more than one radiopharmaceutical, may be helpful for disease characterization. Figures 7 shows a man with concurrent nasopharyngeal cancer and prostate adenocarcinoma (Gleason 7). While the nasopharyngeal cancer is intensely FDG-avid and mildly PSMA-avid, the prostate cancer is intensely PSMAavid and mildly FDG-avid. It is important to remember that the intensity of PSMA uptake does not imply a diagnosis of prostate versus non-prostatic malignancy. Figure 8 shows a case of an intensely PSMA-avid sternal metastasis related to prostate cancer in a patient with an intensely PSMA-avid hepatocellular carcinoma. Additional non-prostatic malignancies that have been shown to be PSMA-avid include malignancies of the gastrointestinal tract, renal cell carcinoma, neuroendocrine malignancy, gliomas and breast cancer, among others. Further, as our experience with PSMA-targeted PET increases, so too does our appreciation of PSMA uptake due to benign etiologies (Figure 9 and 10) and unusual patterns of prostate cancer disease spread (Figure 11). Ultimately, it is important to have the complete patient history, including any prior imaging prior to PSMA-targeted PET interpretation and even so, biopsy may be needed for pathologic characterization in certain cases.

\section{Clinical indications for PSAM-targeted PET}

While access to PSMA-targeted PET currently remains variable across Canada, Table 3 provides suggested indications including the quality of supportive evidence and recommendation strength [16]. 


\section{Staging of biochemical recurrence}

The most common indication for PSMA-targeted PET is for the evaluation of disease in men with biochemical failure after primary prostate cancer therapy. The clinical management of men with biochemical recurrence depends on disease extent: local recurrence (Figure 12) or local recurrence with regional nodal metastases versus distant metastatic disease (Figure 13). While discussion regarding optimal management of men with biochemical failure is ongoing, there is general agreement that accurate identification of disease location and extent is key to determining the most appropriate management (Figure 14). Several trials have evaluated the role of surgery, radiation or a combination of ablative procedures, in men with localized, oligometastatic or low-volume disease [17-20]. Further, studies have shown high performance of PSMA-targeted PET in this patient population.

There is a correlation between observed disease detection rates and serum PSA level [2124], although PSMA-targeted PET may be useful in detecting disease sites even when the serum PSA is low. Approximately 38\% of PSMA-targeted PET scans show disease sites in men with PSA $<0.5 \mathrm{ng} / \mathrm{ml}$ [25]. A prospective trial of 635 patients showed a positive predictive value of $84-92 \%$ for ${ }^{68}$ Ga-PSMA-11 PET in localizing disease, with PET-directed focal therapy alone leading to a PSA drop of $\geq 50 \%$ in $31 / 39(80 \%)$ patients [26]. A literature review suggested the impact of PSMA-targeted PET on management ranged from $30 \%$ to $76 \%$ among 1346 patients. Modifications made to pre- PSMA-targeted PET planned management included avoidance of systemic therapy (19-50\%) and PET-directed local therapy in up to 60\% of cases [25]. Notably, prospective series of men with early biochemical failure and negative PSMA-targeted PET may still have high rates of response to salvage radiotherapy suggesting these negative scans may indicate subclinical disease within the prostate bed rather than subclinical metastatic disease $[27,28]$. Several prospective trials and long-term outcome studies on the impact of PSMAtargeted PET-directed personalized patient management for prostate cancer are underway (i.e., NCT03582774 - PSMA-SRT Trial, NCT04557501 - PATRON and NCT03525288 - PSMAPETgRT)

Despite the strengths of PSMA-targeted PET, there are also limitations and pitfalls (Figure 15). Reasons for false negative studies include, among others: 1. Small tumor volume such as may be seen in men with early stage biochemical recurrence when the serum PSA is below $0.5 \mathrm{ng} / \mathrm{ml}$ or 2 . Neuroendocrine differentiation of prostate cancer with downregulation of PSMA expression [29]. It is estimated neuroendocrine differentiation occurs in $5-10 \%$ of prostate cancers overall and approximately $30 \%$ of men with advanced disease [30]. Androgen receptor inhibition has been shown to increase PSMA expression in prostate cancer cells early on (approximately 4 weeks following ADT) [31]. Continuous, long-term ADT likely induces downregulation or loss of androgen receptors and PSMA expression in addition to decreasing tumour volume $[32,33]$. Thus, short-term ADT likely increases PSMA-targeted PET positivity 
whereas prolonged continuous ADT is associated with a higher likelihood of a negative PSMAtargeted PET.

\section{Primary staging of high-risk prostate cancer}

Studies assessing the use of PSMA-targeted PET for staging men with prostate cancer are not numerous, however, there is a growing body of supportive literature in patients with high-risk disease. The prospective, multi-center randomized Pro-PSMA trial assessed whether PSMAtargeted PET/CT could replace CT and bone scintigraphy (conventional imaging = $\mathrm{CI}$ ) in the initial staging of high-risk localized prostate cancer. There were 302 men randomized to CI or PET/CT. PET/CT performed significantly better than CI with sensitivity, specificity and overall accuracy of PET/CT versus CI of $85 \%$ versus $38 \%, 98 \%$ versus $91 \%$, and $92 \%$ versus $65 \%$, respectively. Furthermore, there were less equivocal findings on PET/CT than on CI (7\% versus $23 \%$ ) [34]. Although the sensitivity of PET/CT in detecting nodal disease may not be enough to obviate the need for lymph node dissection in patients at risk for disease spread, PET/CT may identify nodes outside of the routine surgical field as well as additional sites of distant disease (Figure 16). In the Pro-PSMA trial, PSMA-targeted PET/CT resulted in more frequent management change than CI (28\% vs $15 \%)$ and when looking at patients who had less than 4 metastases on CI, a change in management occurred with subsequent PET/CT in more than 1 in 4 men [34]. To date, comparisons of outcome using PET/CT versus CI remain scarce.

\section{Primary detection of tumor as an adjunct to multiparametric MRI}

The current workup of patients with clinically suspected prostate cancer includes transrectal ultrasound (TRUS)-guided systematic biopsy. TRUS-guided biopsies are associated with a relatively high false negative rate, with clinically significant prostate cancer missed in approximately a quarter of men $[35,36]$. This may be due to constraints of random samples, or technical limitations in obtaining samples from specific areas such as the fibromuscular stroma, apex, or midline gland $[37,38]$. In recent years, multiparametric magnetic resonance imaging (mpMR) has been incorporated in the workup of men with suspected prostate cancer showing considerable promise in detection, localization, risk stratification and staging of prostate cancer. In a meta-analysis of 7 studies evaluating mpMR, de Rooij et al. found a pooled sensitivity and specificity of 0.74 and 0.88 , respectively, with negative predictive value of 0.65-0.94 [39]. mpMR is reported using a standardized scheme (PI-RADS - Prostate Imaging and Reporting Archiving Data System). A validation study of lesions with score $\geq 4$ (using PI-RADS v.2 suspicious for malignancy) found an overall sensitivity and specificity of 77.0-77.3\% and 71.4$73.8 \%$, respectively, for the detection of castration-sensitive prostate cancer [40]. A small series suggested PSMA-targeted PET identified histologically confirmed intraprostatic cancer sites [41] and may have performance comparable and complementary to mpMRI in the characterization of clinically significant (Gleason Grade Group $\geq 2$ ) disease [42]. The role of PSMA-targeted PET as an adjunct to mpMR for primary detection of clinically significant prostate cancer, especially 
lesions with a PI-RADS v. 2 score of 3 (equivocal) or 4 (suspicious) is not well established and is being investigated in a prospective trial [NCT03149861] (Figure 17).

\section{Evaluation of men with metastatic prostate cancer}

Currently, clinical management pathways for men with metastatic prostate cancer are based on the results of conventional imaging with a bone scan and CT. PSMA-targeted PET can identify sites of metastatic disease at the time of initial staging, biochemical recurrence and in the setting of castrationresistance, in the absence of lesions on conventional imaging [43]. Although the impact of PSMA-targeted PET on management and outcome is a topic of research, in general, accurate identification of metastatic disease is important to determining appropriate management. For example, in the setting of hormone sensitive metastatic disease, the extent of disease is used to inform decisions on the addition of chemotherapy or second line hormone therapy to standard androgen deprivation with LHRH agonists or antagonists [44]. In the setting of oligometastatic/ oligoprogressive metastatic prostate cancer, small Phase II randomized trials $[45,46]$ have suggested a benefit of deferring initiation of or changes in systemic therapy. Additional data from large randomized trials such as the PLATON study (CCTG PR20 NCT03784755) is being obtained.

Currently, the established response criteria across the spectrum of prostate cancer, including castrationresistant disease are based on clinical, biochemical, and conventional imaging parameters [47]. While PET based biomarkers offer the potential for quantitative evaluation of response, these metrics have yet to be validated in clinical populations and are not yet part of current decision-making pathways [48]. While the clinical importance of more comprehensive staging and PET based response assessment remains to be defined, PSMAtargeted PET may prove to be increasingly helpful as a diagnostic tool paired with PSMAtargeted therapy. Both ${ }^{177} \mathrm{Lu}$ and ${ }^{225} \mathrm{Ac}$ PSMA-targeted radioligand therapy is emerging as a promising therapy for castrationresistant disease [49]. Recently, several clinical trials have assessed the role of radionuclide therapy using ${ }^{177} \mathrm{Lu}$-labeled PSMA ligands in men with metastatic castration-resistant prostate cancer after failure of androgen receptor axis targets (enzalutamide/ abiraterone) and/or chemotherapy. The European Association of Nuclear Medicine guiding principles for radionuclide therapy with ${ }^{177}$ Lu-labeled PSMA-ligands [50] outlines potential indications and procedure guidelines for the use of ${ }^{177}$ Lu-PSMA therapy in men with metastatic castration-resistant prostate cancer. The idea is based on the concept of using a predictive biomarker, specifically high avidity in putative sites of disease on PSMAtargeted PET, followed by individualized treatment with a therapeutic agent such as ${ }^{177} \mathrm{Lu}$ labeled PSMA-targeted compounds [51, 52]. The optimal threshold of PSMA uptake at tumor sites for patient selection prior to radionuclide therapy is a topic of debate with proponent for uptake comparable to liver activity through proponents of intense uptake on the order of SUVmax 20 or higher [50-52]. Regardless, PSMA-targeted PET is likely to prove helpful for determining patient suitability for PSMA-targeted radionuclide therapy. Some authors suggest a 
combination of FDG and PSMA-targeted PET may be preferable to PSMA-targeted PET alone for optimal patient selection. It is thought that patients with PSMA positive and FDG negative disease may have the highest response rates; however, this remains to be verified in clinical trials [49]. Also, the optimal schedule and dose of ${ }^{177}$ Lu-PSMA radiopharmaceuticals remain to be determined and the use of ${ }^{177} \mathrm{Lu}$-PSMA versus ${ }^{225}$ Ac-PSMA radiopharmaceuticals are areas of active investigation. Finally, the results of randomized trials of PSMA-targeted radiopharmaceuticals compared to best supportive care (VISION, NCT03511664), chemotherapy (TheraP, NCT03392428) and enzalutamide (ARROW, NCT03939689) will likely provide additional guidance in the near future.

\section{Current recommendations for PSMA PET/CT in the Canadian context}

Currently, PSMA-targeted PET is being integrated into the prostate cancer care pathway worldwide. While there are no PSMA-targeted PET radiopharmaceuticals approved by Health Canada for routine use in clinical care, it seems reasonable PSMA-targeted PET will become part of routine clinical care in Canada in the near future.

To date, data strongly suggests the performance of PSMA-targeted PET is superior to conventional imaging in characterizing prostate cancer across the spectrum of disease, although the ultimate impact of this on patient outcome remains to be determined. As summarized in Table 3, PSMA-targeted PET is useful primarily for staging primary high-risk prostate cancer and restaging biochemically recurrent disease after primary therapy. To expand further, patients with high-risk or very high-risk prostate cancer in whom imaging with conventional modalities fail to demonstrated metastatic disease (and where its identification would lead to a treatment change), PSMA-targeted PET may be contemplated. In those with non-metastatic castrationresistant prostate cancer in whom PSA kinetics are favourable and where systemic treatment intensification is not warranted, identification of metastases by PSMA-targeted PET may allow for inclusion into clinical trials assessing utility of metastasis directed therapy.

A large Canadian clinical trial of PSMA-targeted PET informed treatment versus standard of care informed treatment in high-risk primary and recurrent disease is planned (i.e. PATRON - NCT04557501). In the setting of established metastatic disease (castration-sensitive and castration-resistant) the utility of PSMA-targeted PET is less well established, although it will likely present a powerful tool, particularly when paired with PSMA-targeted radiopharmaceutical therapy.

Despite the superior detection of disease by PSMA-targeted PET, it must be emphasized that to date, there is no evidence to support such enhancements have led to improved clinical outcomes. Clinical trials addressing this issue are underway, and thus, while today care pathways remain based on well established clinical criteria and standard of care imaging that does not include PSMA-targeted PET, this is likely to change in the near future. 


\section{Conclusions}

PSMA-targeted PET has high overall accuracy for the detection of prostate cancer and a growing role in determining patient management, particularly in men with high-risk disease at the time of staging and biochemical failure after primary therapy. As PSMA-targeted PET becomes more ubiquitous in clinical practice, increased familiarization with potential pitfalls in image interpretation and knowledge of the reasons for both false positive and false negative examinations are critical. Ongoing research will help to clarify the long-term impact of PSMAtargeted PET on patient outcome as well as to better define the relative strengths and weakness of the individual available PSMA-targeted PET radiopharmaceuticals. 


\section{References}

1. Maurer T, Eiber M, Schwaiger M, Gschwend JE. Current use of PSMA-PET in prostate cancer management. Nat Rev Urol 2016;13:226-235.

2. Silver DA, Pellicer, I, Fair WR, Heston WD, Cordon-Cardo C. Prostate-specific membrane antigen expression in normal and malignant human tissues. Clin Cancer Res 1997;3(1):81-85.

3. Kasperzyk JL, Finn SP, Flavin R, et al. Prostate-specific membrane antigen protein expression in tumor tissue and risk of lethal prostate cancer. Cancer Epidemiol Biomarkers Prev 2013;22(12):2354-2363.

4. Meller BF, Bremmer CO, Sahlmann S, et al. Alterations in androgen deprivation enhanced prostate-specific membrane antigen (PSMA) expression in prostate cancer cells as a target for diagnostics and therapy. EJNMMI Res 2015;5(1):66.

5. Hillier SM, Maresca, KP, Femia FJ, et al. Preclinical evaluation of novel glutamate-urealysine analogues that target prostate-specific membrane antigen as molecular imaging pharmaceuticals for prostate cancer. Cancer Res 2009;69(17):6932-6940.

6. Chen Y, Pullambhatla M, Foss CA, et al. 2-(3-\{1-Carboxy-5-[(6-[18F]fluoro-pyridine-3carbonyl)-amino]-pentyl \}-ureido)-pen tanedioic acid, [18F]DCFPyL, a PSMA-based PET imaging agent for prostate cancer. Clin Cancer Res 2011;17(24):7645-7653.

7. Haupt F, Dijkstra L, Alberts I. ${ }^{68} \mathrm{Ga}-\mathrm{PSMA}-11$ PET/CT in patients with recurrent prostate cancer-a modified protocol compared with the common protocol. Eur J Nucl Med Mol Imaging 2020;47(3):624-631.

8. Mena E, Lindenberg ML, Turkbey IB, et al. ${ }^{18}$ F-DCFPyL PET/CT Imaging in Patients with Biochemical Recurrence Prostate Cancer after Primary Local Therapy. J Nucl Med 2020;61(6):881-889.

9. Giesel FL, Knorr K, Spohn F, et al. Detection Efficacy of ${ }^{18}$ F-PSMA-1007 PET/CT in 251 Patients with Biochemical Recurrence of Prostate Cancer After Radical Prostatectomy. J Nucl Med 2019;60(3):362-368.

10. Delso G, Fürst S, Jakoby B, et al. Performance measurements of the Siemens mMR integrated whole-body PET/MR scanner. J Nucl Med 2011;52:1914-1922.

11. Metser U, Chan R, Veit-Haibach P, Ghai S, Tau N. Comparison of MRI Sequences in Whole-Body PET/MRI for Staging of Patients With High-Risk Prostate Cancer. AJR Am J Roentgenol 2019;212:377-381.

12. Demirici E, Sahin OE, Ocak M, Akovali B, Nematyazar J, Kabasakal L. Normal Distribution Pattern and Physiological Variants of 68Ga-PSMA-11 PET/CT Imaging. Nucl Med Commun 2016;27(11):1169-1979.

13. Giesel FL, Hadashchik B, Cardinale J, et al. F-18 labelled PSMA-1007: biodistribution, radiation dosimetry and histopathological validation of tumor lesions in prostate cancer patients. Eur J Nucl Med Mol Imaging 2017;44(4):678-688.

14. Barbosa FG, Querioz MA, Nunes RF, et al. Nonprostatic diseases on PSMA PET imaging: a spectrum of benign and malignant findings. Cancer Imaging 2020;20(1):23.

15. Noto B, Vrachimis A, Schäfers M, et al. Subacute stroke mimicking cerebral metastasis in 68Ga-PSMA-HBED-CC PET/CT. Clin Nucl Med 2016;41:e449-e451.

16. Trabulsi EJ, Rumble RB, Jadvar H, et al. Optimum imaging strategies for advanced prostate cancer: ASCO guideline. J Clin Oncol 2020;38(17):1963-1996. 
17. Radwan N, Phillips R, Ross A, et al. A Phase II Randomized Trial of Observation Versus Stereotactic Ablative RadiatIon for OLigometastatic Prostate CancEr (ORIOLE). BMC Cancer 2017;17(1):453.

18. Parker CC, James ND, Brawley CD, et al. Radiotherapy to the primary tumour for newly diagnosed, metastatic prostate cancer (STAMPEDE): a randomised controlled phase 3 trial. Lancet 2018;392:2353-2366.

19. Jenjitranant P, Touijer KA. Role of surgery in oligometastatic prostate cancer. Prostate Int 2019;7(4):125-130.

20. Decaestecker K, De Meerleer G, Ameye F, et al. Surveillance or metastasis-directed Therapy for OligoMetastatic Prostate cancer recurrence (STOMP): study protocol for a randomized phase II trial. BMC Cancer.2014;14:671.

21. Han S, Woo S, Kim YJ, Suh CH. Impact of ${ }^{68}$ Ga-PSMA PET on the management of patients with prostate cancer: a systematic review and meta-analysis. Eur Urol 2018;74(2):179-190.

22. Perera M, Papa N, Christidis D, et al. Sensitivity, specificity, and predictors of positive ${ }^{68} \mathrm{Ga}$-prostate-specific membrane antigen positron emission tomography in advanced prostate cancer: a systematic review and meta-analysis. Eur Urol 2016;70(6):926-937.

23. von Eyben FE, Picchio M, von Eyben R, Rhee H, Bauman G. ${ }^{68} \mathrm{Ga}$-labeled prostatespecific membrane antigen ligand positron emission tomography/computed tomography for prostate cancer: a systematic review and meta-analysis. Eur Urol Focus 2018;4(5):686-693.

24. Afshar-Oromieh A, Holland-Letz T, Giesel FL, et al. Diagnostic performance of ${ }^{68} \mathrm{Ga}$ PSMA-11 (HBED-CC) PET/CT in patients with recurrent prostate cancer: evaluation in 1007 patients. Eur J Nucl Med Mol Imaging 2017;44(8):1258-1268.

25. Ekmekcioglu O, Busstra M, Klass ND, Verzijlbergen F. Bridging the imaging gap: PSMA PET/CT has a high impact on treatment planning in prostate cancer patients with biochemical recurrence - a narrative review of literature. J Nucl Med 2019;60(10):13941398.

26. Fendler WP, Calais J, Eiber M, et al. Assessment of ${ }^{68}$ Ga-PSMA-11 PET Accuracy in Localizing Recurrent Prostate Cancer. JAMA Oncol 2019;5(6):856-863.

27. Luiting HB, van Leeuwen PJ, Busstra MB, et al. Use of gallium-68 prostate-specific membrane antigen positron-emission tomography for detecting lymph node metastases in primary and recurrent prostate cancer and location of recurrence after radial prostatectomy: an overview of the current literature. BJU Int 2020;125(2):206-214.

28. Emmett L, van Leeuwen PJ, Nandurkar R, et al. Treatment outcomes from ${ }^{68} \mathrm{Ga}-\mathrm{PSMA}$ PET/CT-informed salvaged radiation treatment in men with rising PSA after radial prostatectomy: prognostic value of a negative PSMA PET. $J$ Nucl Med 2017;58(12):1972-1976.

29. Tosoian JJ, Gorin MA, Rowe SP, et al. Correlation of PSMA-targeted 18F-DCFPyL $\mathrm{PET} / \mathrm{CT}$ findings with immunohisotochemical and genomic data in patient with metastatic neuroendocrine prostate cancer. Clin Genirourin Cancer 2016;15:365-e8.

30. Patel GK, Chugh N, Tripathi M. Neuroendocrine Differentiation of Prostate Cancer - An Intriguing Example of Tumor Evolution at Play. Cancers 2019;11(10):1405. 
31. Hope TA, Truillet C, Ehman EC, et al. 68Ga-PSMA-11 PET Imaging of Response to Androgen Receptor Inhibition: First Human Experience. J Nucl Med 2017;58(10):81-84.

32. Afshar-Oromieh A, Debus N, Uhrig M, et al. Impact of long-term androgen deprivation therapy on PSMA ligand PET/CT in patients with castration-sensitive prostate cancer. Eur J Nucl Med Mol Imaging 2018;45(12):2045-2054.

33. Emmett L, Yin C, Crumbaker M, et al. Rapid Modulation of PSMA Expression by Androgen Deprivation: Serial (68)Ga-PSMA-11 PET in Men with Hormone-Sensitive and Castrate-Resistant Prostate Cancer Commencing Androgen Blockade. J Nucl Med 2019;60(7):950-954.

34. Hofman MS, Lawrentshuk N, Francis RJ, et al. Prostate-specific membrane antigen PET$\mathrm{CT}$ in patients with high-risk prostate cancer before curative-intent surgery or radiotherapy (proPSMA): a prospective, randomised, multicentre study. Lancet 2020;395:1208-1216.

35. Roehl KA, Antenor JA, Catalona WJ. Serial biopsy results in prostate cancer screening study. J Urol 2002;167:2435-2439.

36. Rabbani F, Stroumbakis N, Kava BR, et al. Incidence and clinical significance of falsenegative sextant prostate biopsies. J Urol 1998;159:1247-1250.

37. Fleshner NE, O'Sullivan M, Fair WR. Prevalence and predictors of a positive repeat transrectal ultrasound guided needle biopsy of the prostate. J Urol 1997;158:505-509.

38. Zaytoun OM, Jones JS. Prostate cancer detection after a negative prostate biopsy: lessons learnt in the Cleveland Clinic experience. Int J Urol 2011;18:557-568.

39. de Rooij M, Hamoen EHJ, Fütterer JJ, Barentsz JO, Rovers MM. Accuracy of multiparametric MRI for prostate cancer detection: a meta-analysis. AJR Am J Roentgenol 2014;202(2):343-351.

40. Park SY, Jung DC, Oh YT, et al. Prostate Cancer: PI-RADS Version 2 Helps Preoperatively Predict Clinically Significant Cancers. Radiology 2016;280(1):108-116.

41. Woythal N, Arsenic R, Kempkensteffen C, et al. Immunohistochemical validation of PSMA expression measured by 68Ga-PSMA PET/CT in primary prostate cancer. $J$ Nucl Med 2018;59(2):238-243.

42. Scheltema MJ, Chang JI, Stricker PD, et al. Diagnostic accuracy of ${ }^{68}$ Ga-prostate-specific membrane antigen (PSMA) positron-emission tomography (PET) and multiparametric (mp)MRI to detect intermediate-grade intra-prostatic prostate cancer using whole-mount pathology: impact of the addition of ${ }^{68} \mathrm{Ga}$-PSMA PET to mpMRI. BJU Int 2019;124(Suppl 1):42-29.

43. Hadaschik BA, Weber M, Iravani A, et al. Prostate-specific membrane antigen positronemission tomography (PSMA-PET) in high-risk nonmetastatic castration-resistant prostate cancer (nmCRPC) SPARTAN-like patients (pts) negative by conventional imaging. Eur Urol Suppl 2019;18(1):E698-E699x.

44. Gravis G, Boher JM, Chen YH, et al. Burden of metastatic castration-naive prostate cancer patients, to identify men more likely to benefit from early docetaxel: further analyses of CHAARTED and GETUG-AFU15 studies. Eur Urol 2018;73(6):847-855.

45. Ost P, Reynders D, Decaestecker K, et al. Surveillance or metastasis-directed therapy for oligometastatic prostate cancer recurrence: a prospective, randomized, multicenter phase II trial. J Clin Oncol 2018;36(5):446-453. 
46. Phillips R, Shi WY, Deek M, et al. Outcomes of observation vs stereotactic ablative radiation for oligometastatic prostate cancer: the ORIOLE phase 2 randomized clinical trial. JAMA Oncol 2020;6(5):650-659.

47. Scher HI, Morris MJ, Stadler WM, et al. Trail design and objectives for castrationresistant prostate cancer: updated recommendations from the Prostate Cancer Clinical Trials Working Group 3. J Clin Oncol 2016;34(12):1402-1418.

48. Vapiwala N, Hofman MS, Murphy DG, Williams S, Sweeney C. Strategies for evaluation of novel imaging in prostate cancer: putting the horse back before the cart. J Clin Oncol 2019;37(10):765-769.

49. Iravani A, Violet J, Azad A, Hofman MS. Lutetium-177 prostate-specific membrane antigen (PSMA) theranostics: practical nuances and intricacies. Prostate Cancer Prostatic Dis 2020;23(1):38-52.

50. Kratochwil C, Fendler WP, Eiber M, et al. EANM Procedure Guidelines for Radionuclide Therapy With ${ }^{177}$ Lu-labelled PSMA-ligands ( ${ }^{177}$ Lu-PSMA-RLT). Eur J Nucl Med Mol Imaging 2019;46(12):2536-2544.

51. Herrmann K, Larson SM, Weber WA. Theranostic Concepts: More Than Just a Fashion Trend-Introduction and Overview. J Nucl Med 2017;58(Suppl 2):1S-2S.

52. Hofman MS, Violet J, Hicks RJ, et al. [(177)Lu]-PSMA-617 radionuclide treatment in patients with metastatic castration-resistant prostate cancer (LuPSMA trial): a singlecentre, single-arm, phase 2 study. Lancet Oncol 2018;19:825-833.

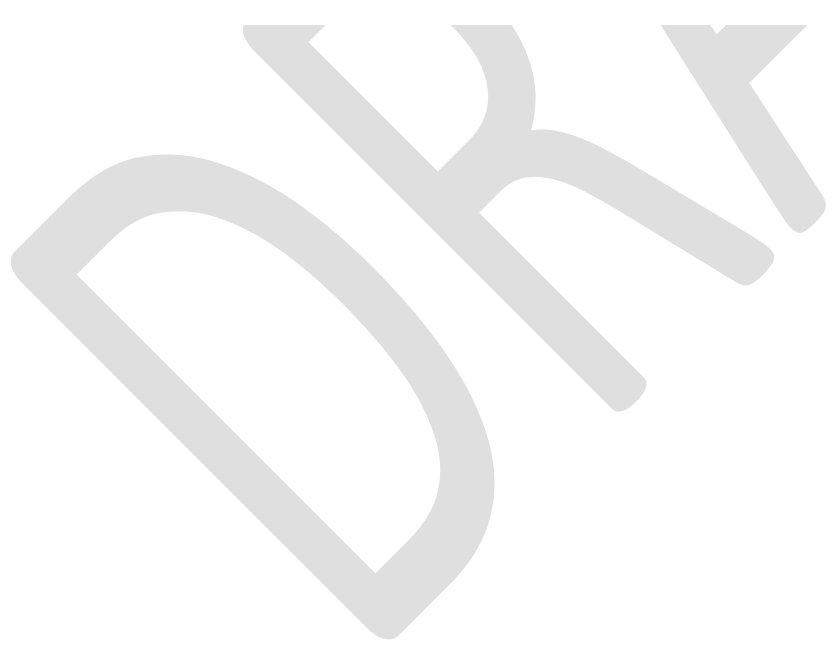




\section{Figures and Tables}

Fig. 1. Normal biodistribution of ${ }^{18} \mathrm{~F}$-DCFPyL (a common PSMA radiopharmaceutical). (A) Maximum Intensity Projection (MIP) image showing high uptake in lacrimal and salivary glands and kidneys (dotted arrow), moderate uptake in liver (solid arrow) and low to moderate uptake in the spleen and vocal cords (arrowhead).

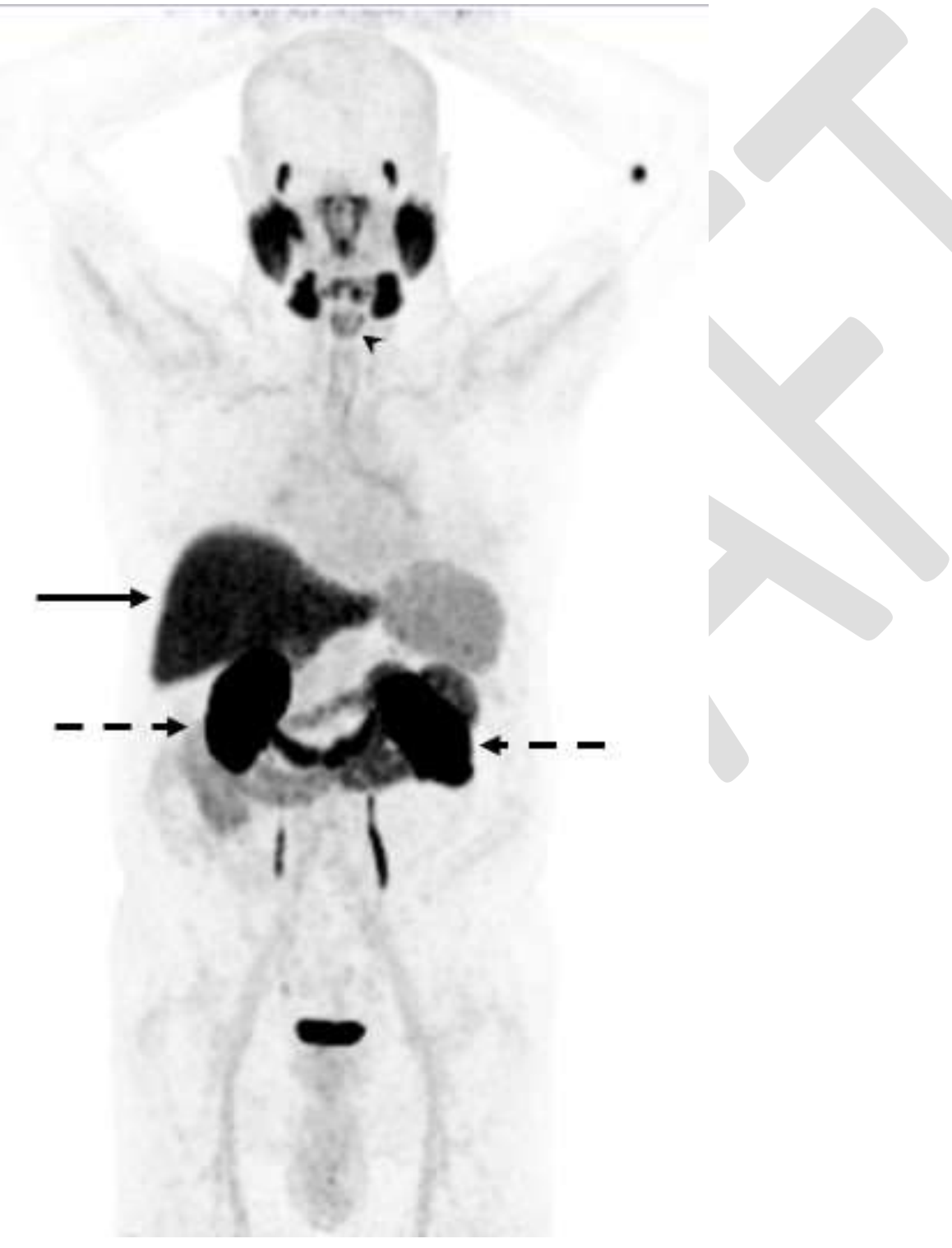


(B) Axial CT, fused ${ }^{18} \mathrm{~F}$-DCFPyL PET/CT and PET images showing high uptake in lacrimal glands (arrow; top row), salivary glands (arrow; middle rows), accessory salivary gland (dotted arrow middle row) and moderate uptake in the larynx (dotted arrow; bottom row).

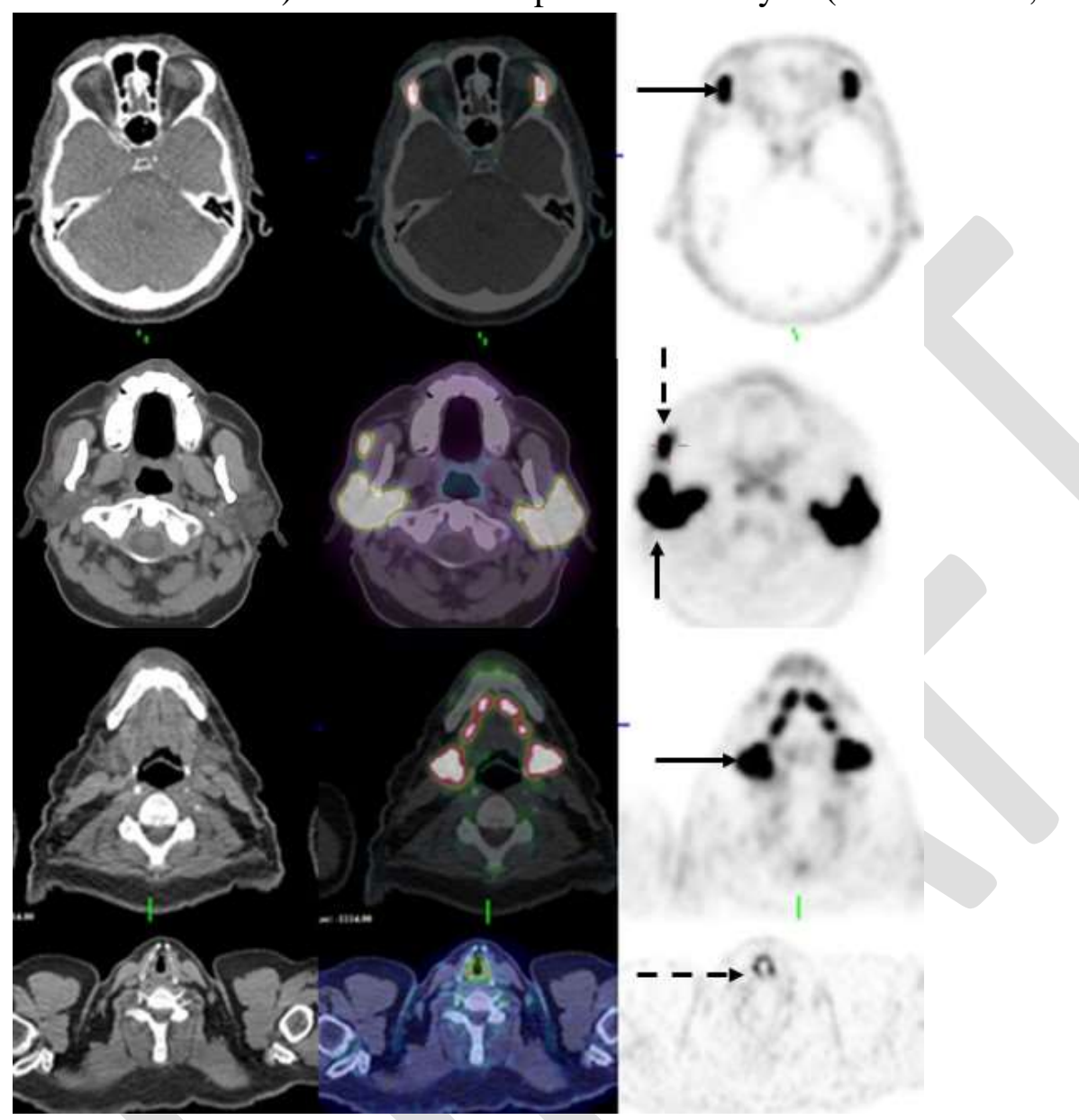


Fig. 2. Axial CT, fused ${ }^{18} \mathrm{~F}$-DCFPyL PET/CT and PET images showing uptake in the stellate ganglia (arrows, top row), celiac ganglia (arrows, middle row) and sacral ganglia (solid arrow bottom row; ureters - dotted arrows).

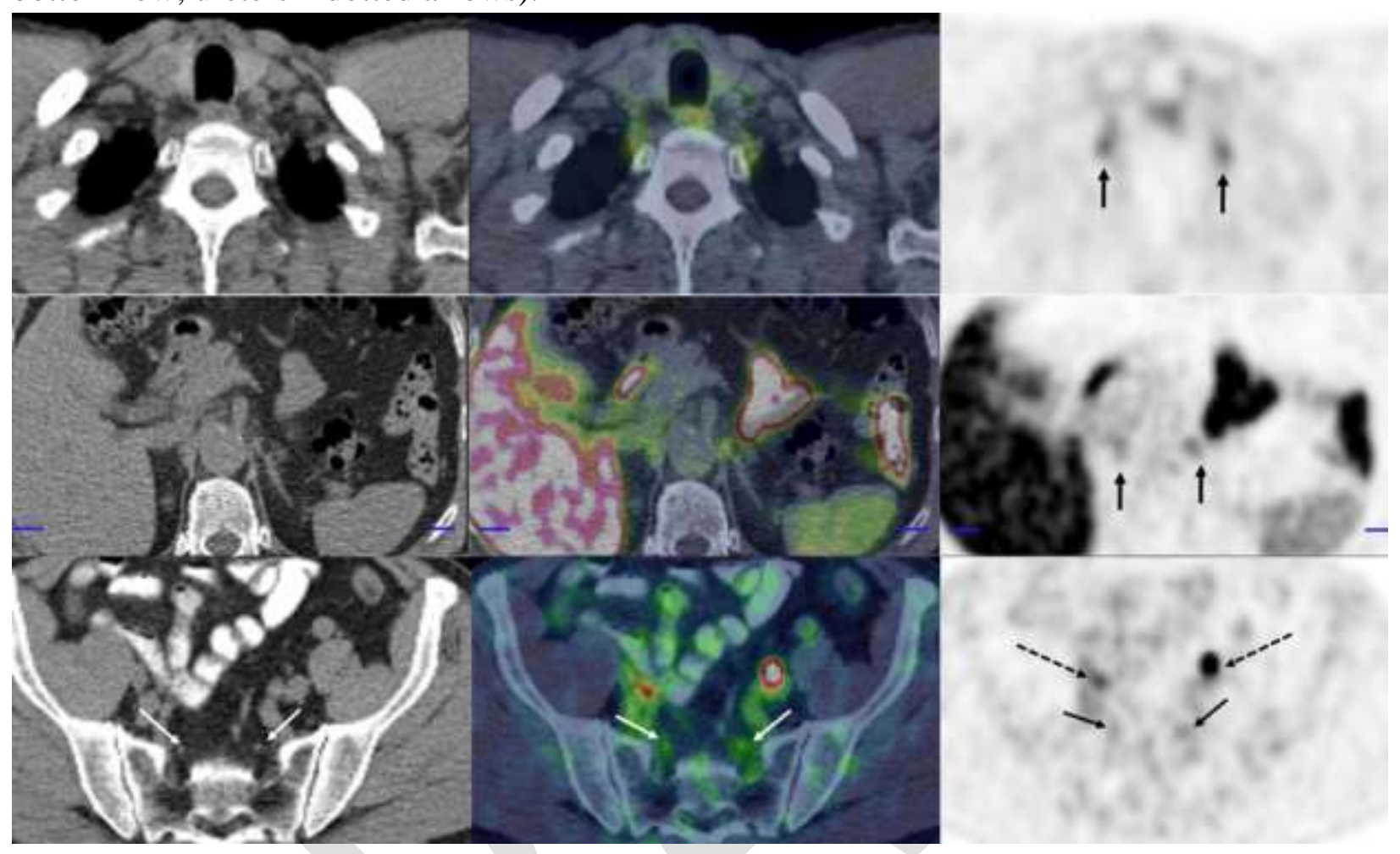


Fig. 3. Benign processes showing increased ${ }^{18} \mathrm{~F}$-DCFPyL uptake on axial PET images, top row and corresponding MRI/ CT images, bottom row. (A) Focal uptake is seen corresponding to a hyperintense left frontotemporal dural-based mass on fluid-attenuated inversion recovery MR image (arrowheads), in keeping with a meningioma. (B) Focal uptake (arrow) is seen in the region of the left basal ganglia with no corresponding abnormality on non-contrast $\mathrm{CT}$. Axial fluid-attenuated inversion recovery MR image (white arrow), shows an infarct. (C) A 76-yearold man with a history of Gleason 7 prostate carcinoma post radical prostatectomy 13 years ago with new biopsy-proven recurrence; PSA $=1.51 \mathrm{ng} / \mathrm{ml}$. Diffuse uptake in patchy ground glass opacities (right upper lobe, dotted arrows: SUVmax, 1.9) and in bilateral hilar and mediastinal lymph nodes (arrowheads; SUVmax, 4.7) is in keeping with an infectious process, likely due to aspiration per clinical history. (D) A 65-year-old man with newly diagnosed cT3b Gleason 9 $(4+5)$ prostate cancer in $5 / 14$ cores. Serum PSA $=7.5 \mathrm{ng} / \mathrm{ml}$. Staging ${ }^{18} \mathrm{~F}$-DCFPyL PET/CT showed uptake in the prostate tumor (not shown) and in a $3.5 \mathrm{~cm}$ (SUVmax, 21.0) enhancing splenic mass (arrow), with imaging features consistent with a hemangioma. (E) A 75-year-old male with a history of pT3bN1 Gleason $4+3$ adenocarcinoma of prostate cancer, with biochemical recurrence post radical prostatectomy (serum PSA $=1.6 \mathrm{ng} / \mathrm{ml}$ ). Sagittal images from a ${ }^{18}$ F-DCFPyL PET/CT shows focal uptake in the L2 vertebral body (arrow), with cortical thickening, coarsening of bone trabeculation and rectangular expansile appearance of the vertebral body, in keeping with Paget's disease.
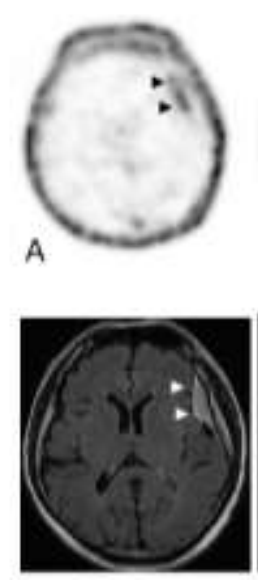
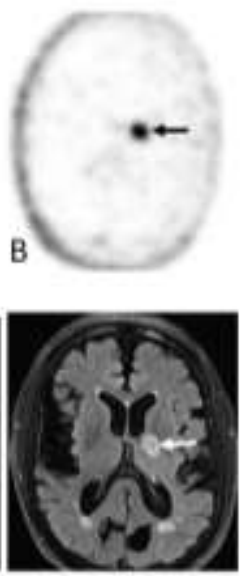
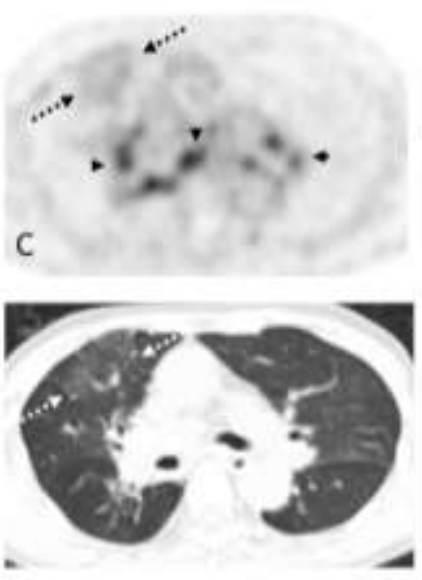
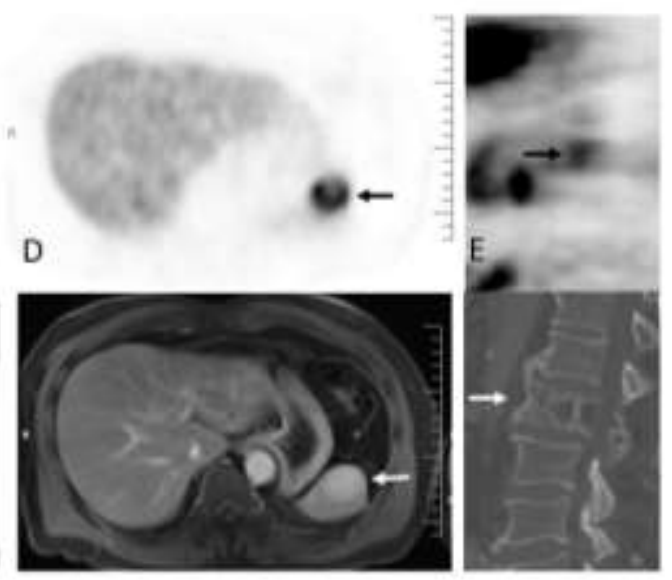
Fig. 4. Axial ${ }^{18}$ F-DCFPyL PET, fused PET/CT and CT images show intense uptake in a patient with a left adrenal leiomyosarcoma.

(A) Intense uptake in the left adrenal gland tumour (arrows).
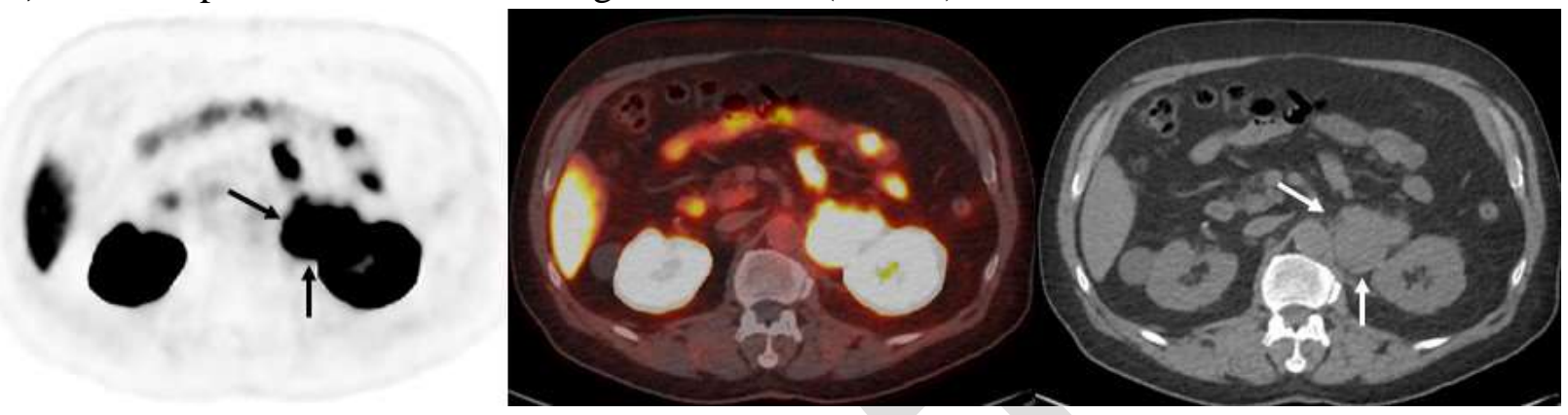

(B) Intense uptake in a lung metastasis (arrows).

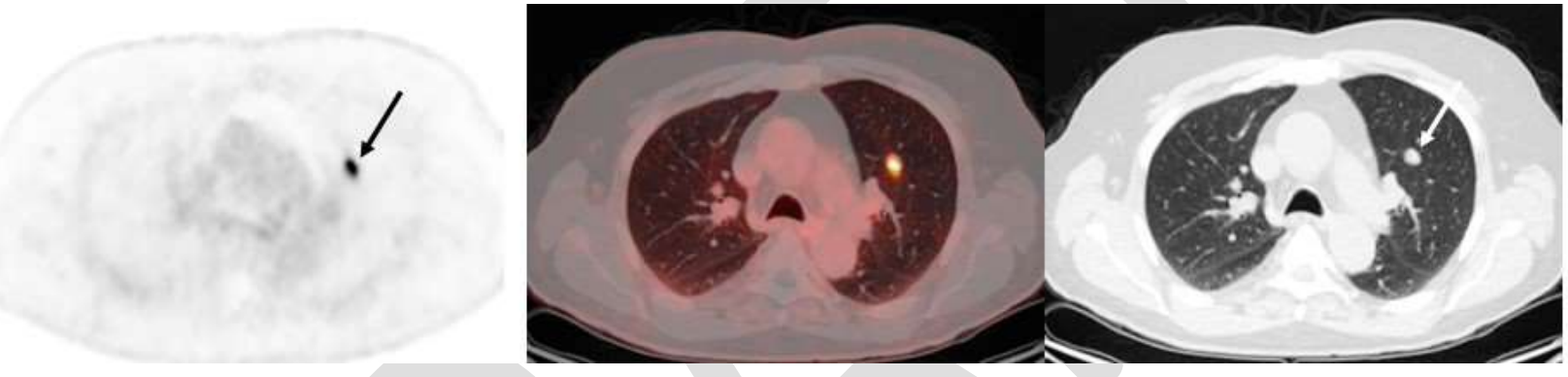

Fig. 5. Axial ${ }^{18}$ F-DCFPyL PET, fused PET/CT and CT images show intense uptake in primary thyroid cancer of the right thyroid lobe (arrow).

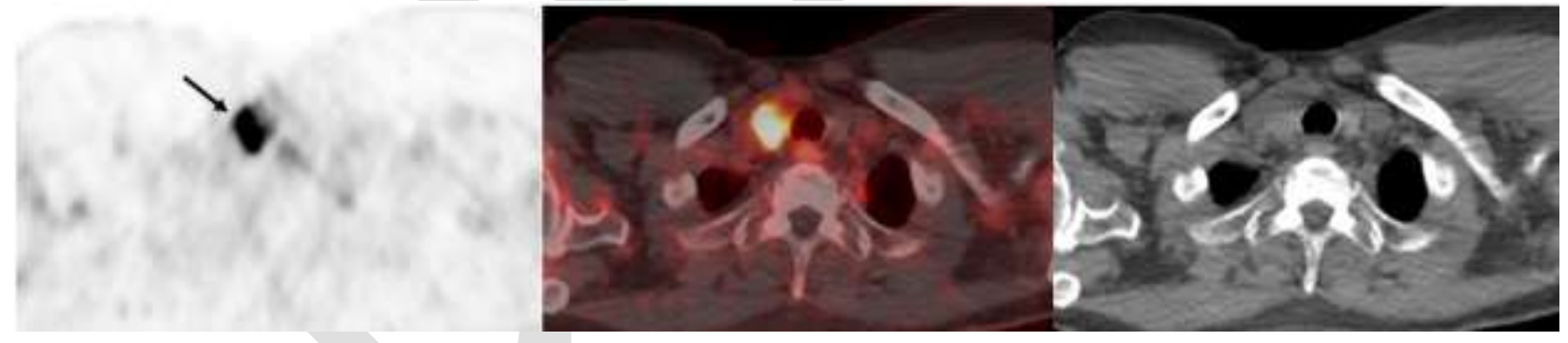

Fig. 6. Axial ${ }^{18} \mathrm{~F}-\mathrm{DCFPyL}$ PET, fused PET/CT and CT images show mild uptake in primary adenocarcinoma of the right lung (arrow).

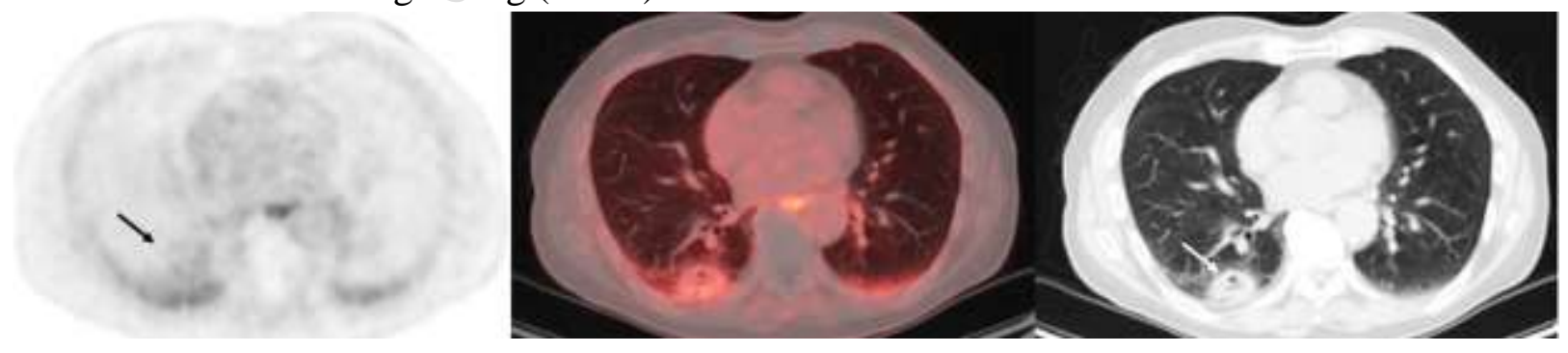


Fig. 7. 58-year-old man with concurrent nasopharyngeal cancer and prostate cancer. (A) Maximum intensity projection image from a ${ }^{18} \mathrm{~F}$-DCFPyL PET/CT shows mild uptake in known nasopharyngeal cancer and intense uptake in known prostate cancer (dotted arrow).

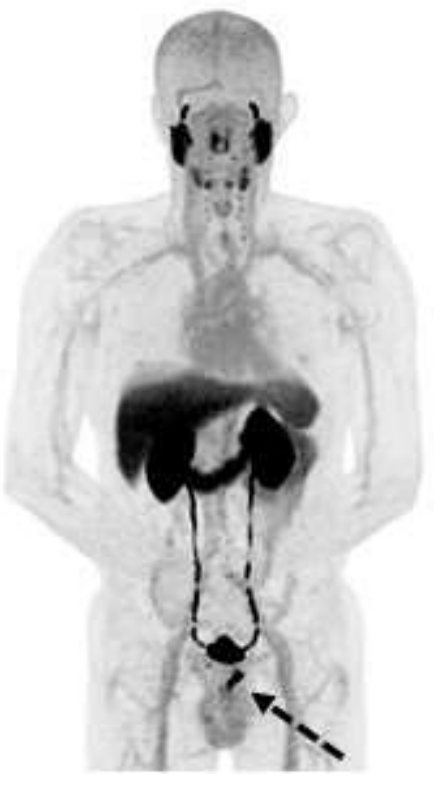

(B) Axial ${ }^{18}$ F-DCFPyL PET, fused PET/CT and CT images confirm mild uptake in known nasopharyngeal cancer (solid arrow top row) and intense uptake in known prostate cancer (dotted arrow bottom row).
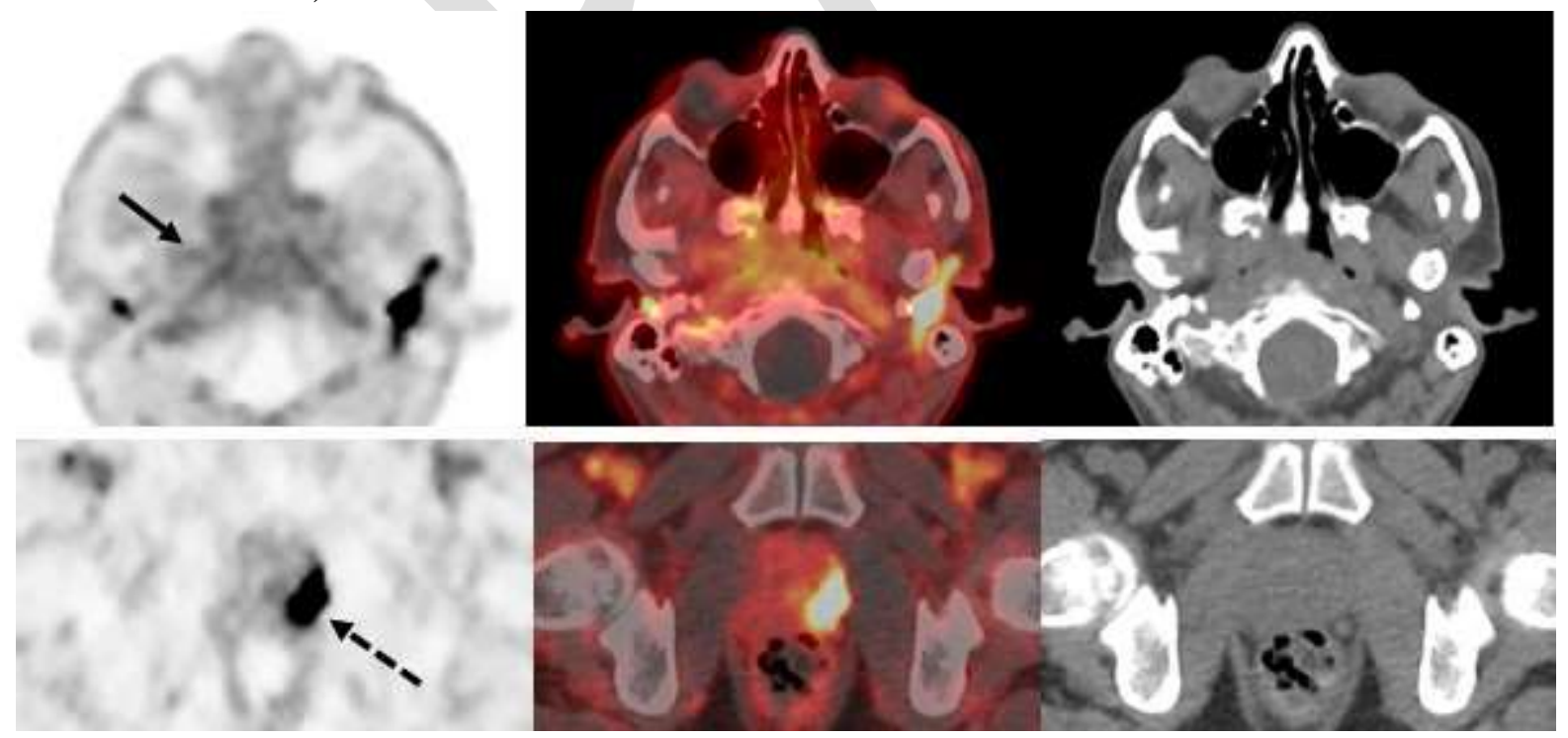
(C) Maximum intensity projection image from a ${ }^{18} \mathrm{~F}-\mathrm{FDG}$ PET/CT shows intense uptake in known nasopharyngeal cancer (solid arrow) and mildly uptake in known prostate cancer (dotted arrow).

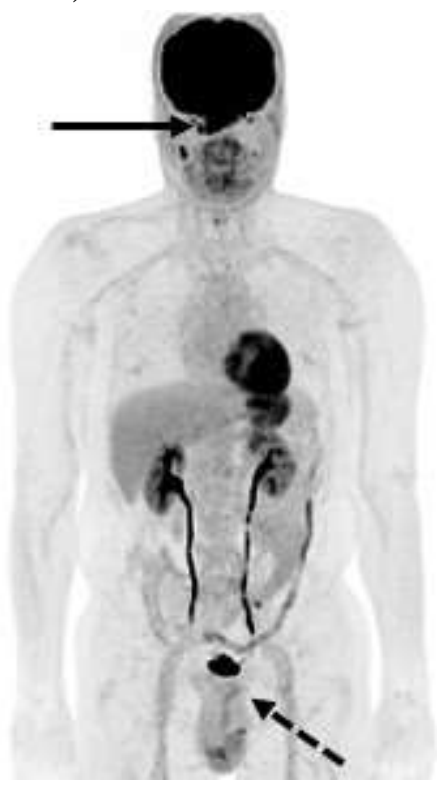

(D) Axial ${ }^{18}$ F-FDG PET, fused PET/CT and CT images show intense uptake in known nasopharyngeal cancer (solid arrow, top row) and a mild uptake in known prostate cancer (dotted arrow; bottom row).
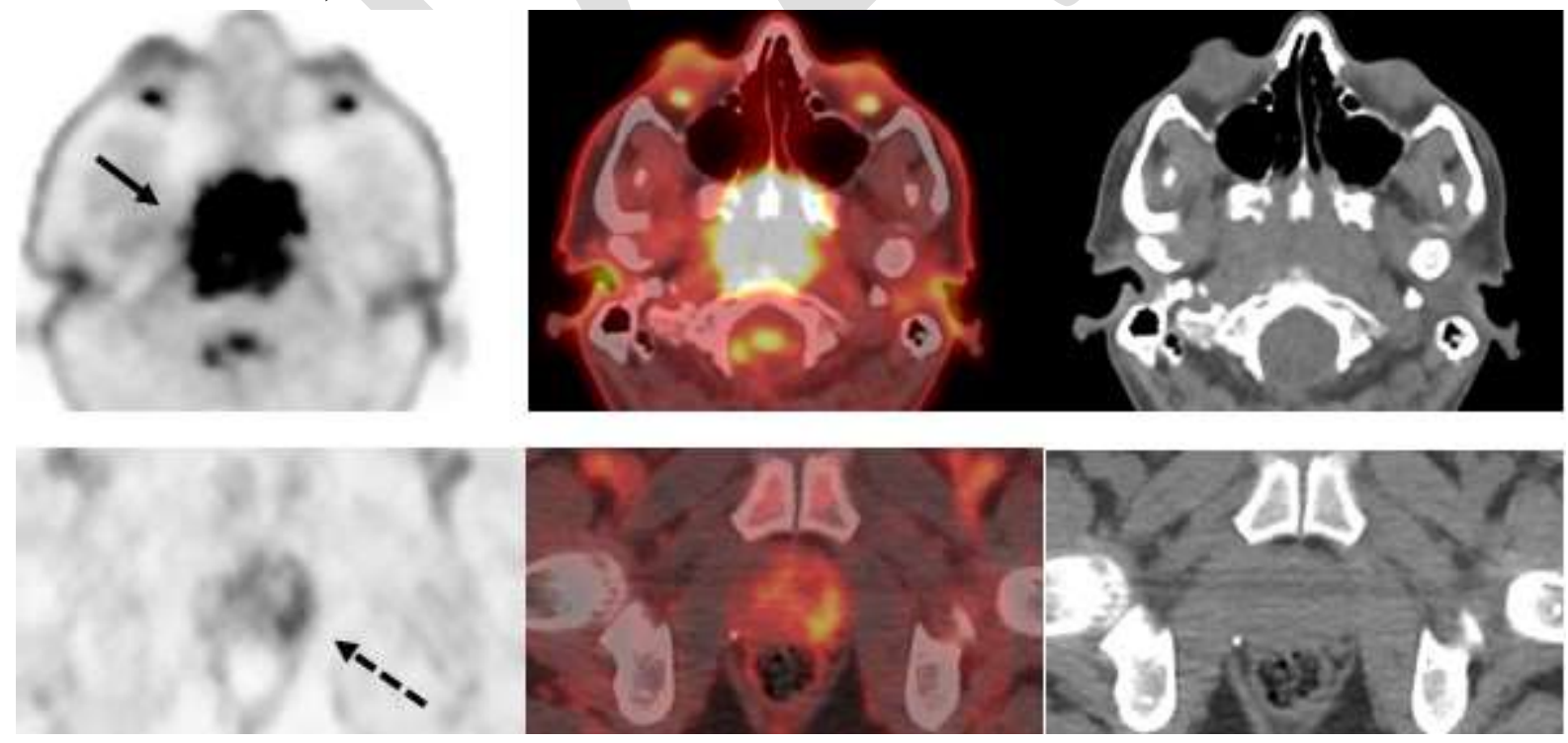
Fig. 8. A 76-year-old man with concurrent hepatocellular carcinoma and prostate cancer. (A) Maximum intensity projection image from a ${ }^{18} \mathrm{~F}$-DCFPyL PET/CT shows intense uptake in hepatocellular carcinoma (solid arrow), prostate cancer (dotted arrow) and a rib metastasis (arrowhead).

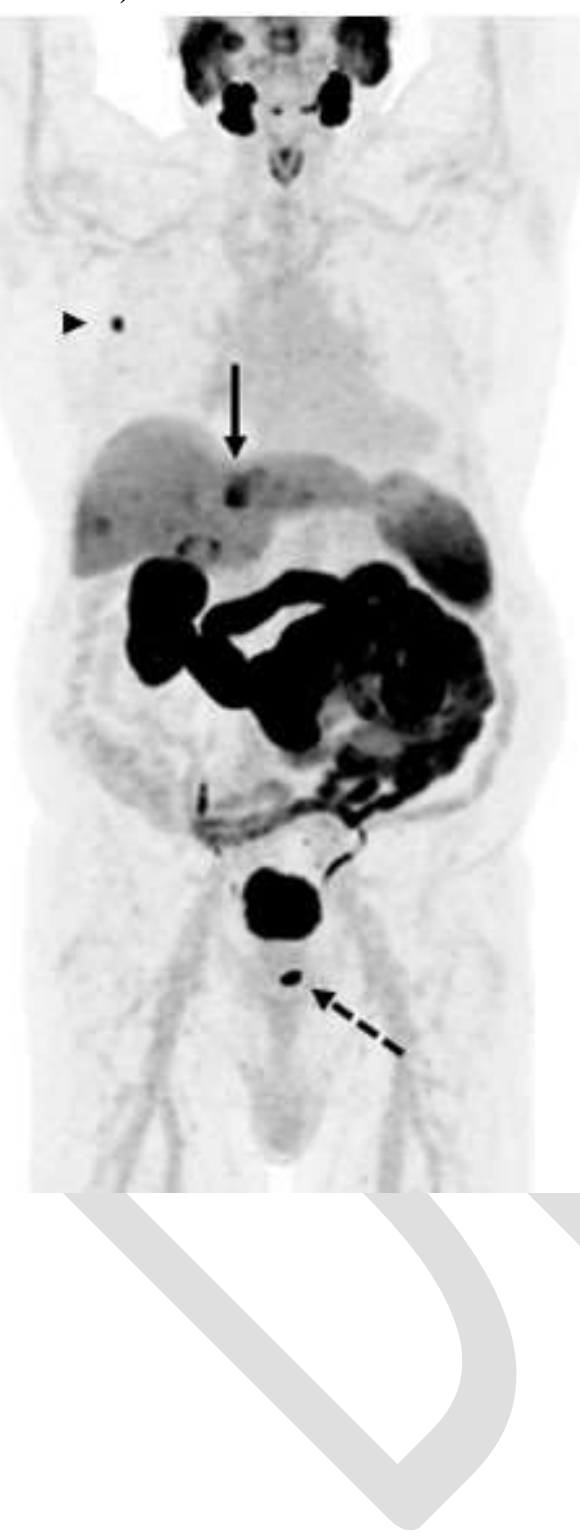


(B) Axial ${ }^{18} \mathrm{~F}-\mathrm{DCFPyL}$ PET, fused PET/CT and CT images show intense uptake in hepatocellular carcinoma (arrows, top row) and prostate cancer (dotted arrow bottom row).
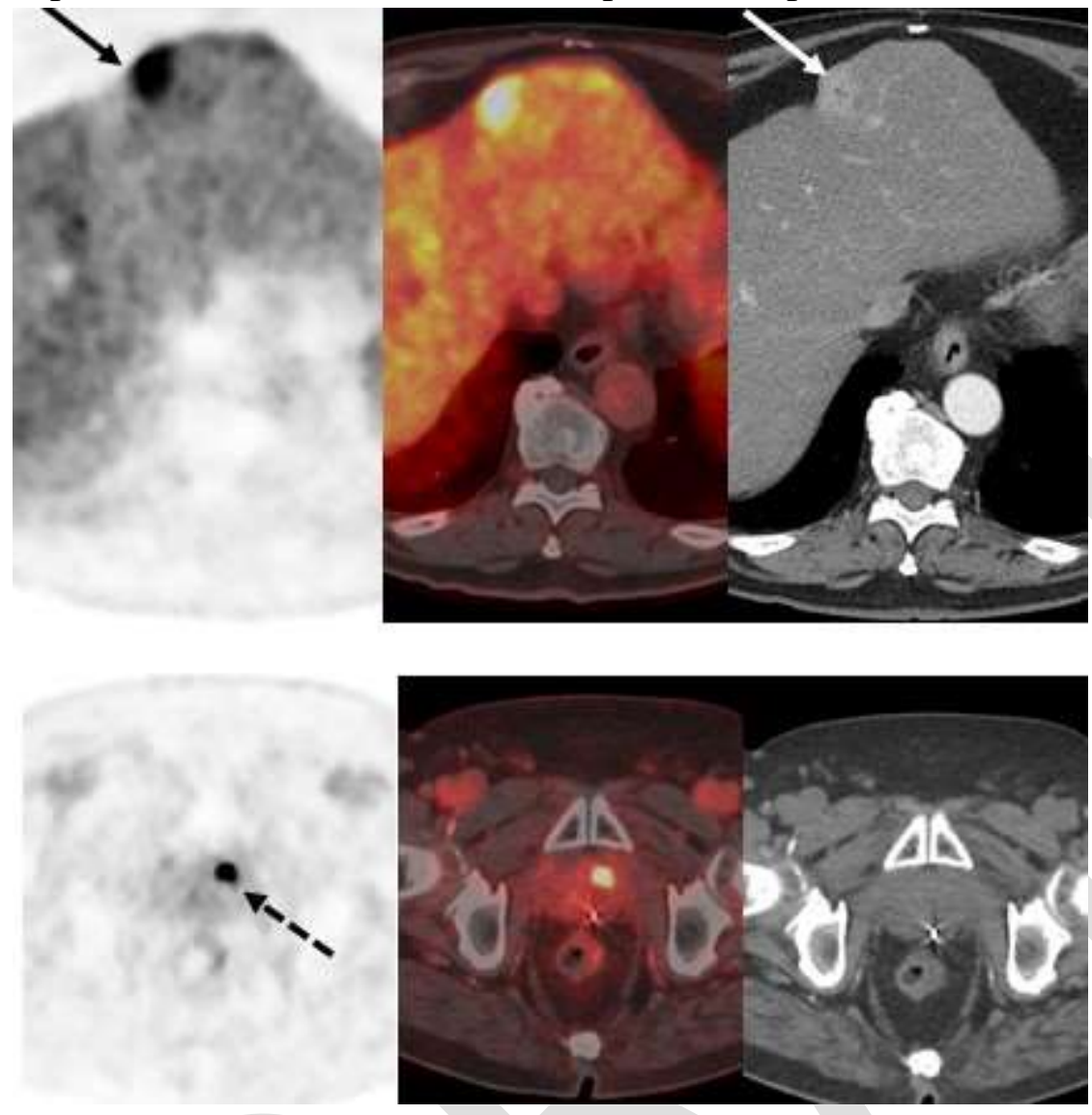
Fig. 9. A 79-year-old man with prostate cancer.

(A) Maximum intensity projection image from a ${ }^{18} \mathrm{~F}-\mathrm{DCFPyL}$ PET/CT shows intense uptake at a site of prostate cancer metastasis to the sternum (arrow). Note the mild uptake in a hepatic lesion.

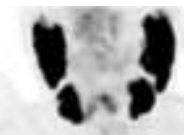

(B) Axial ${ }^{18}$ F-DCFPyL PET, fused PET/CT and CT images show the mild uptake in a hepatic nodule found to be a cavernous hemangioma on MR (not shown).

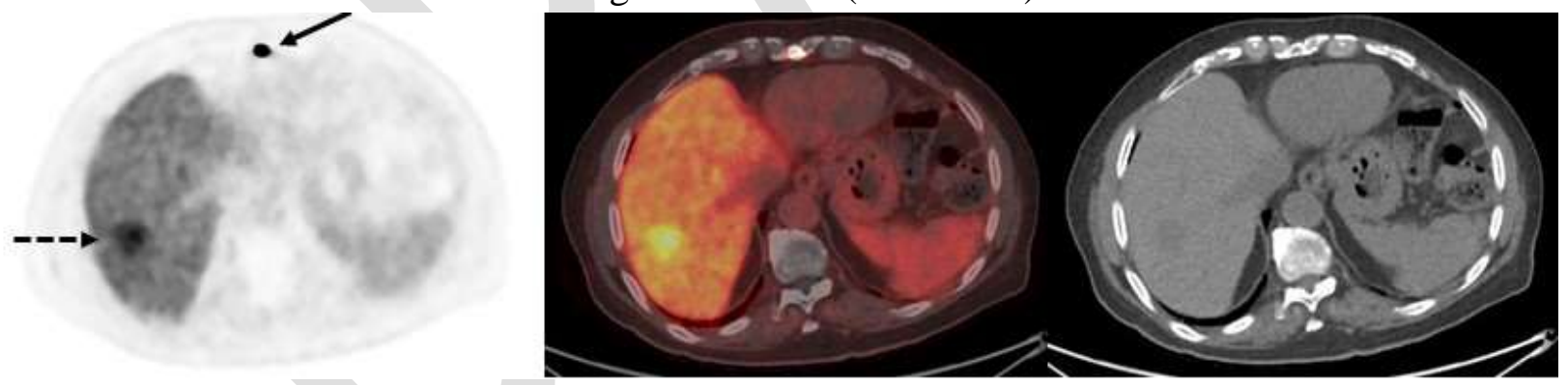


Fig. 10. ${ }^{18} \mathrm{~F}-\mathrm{DCFPyL}$ PET/CT in benign and malignant bone lesions.

(A) Axial ${ }^{18} \mathrm{~F}$-DCFPyL PET, fused PET/CT and CT images show mild uptake associated with Paget's of the right hemipelvis (dotted arrow).
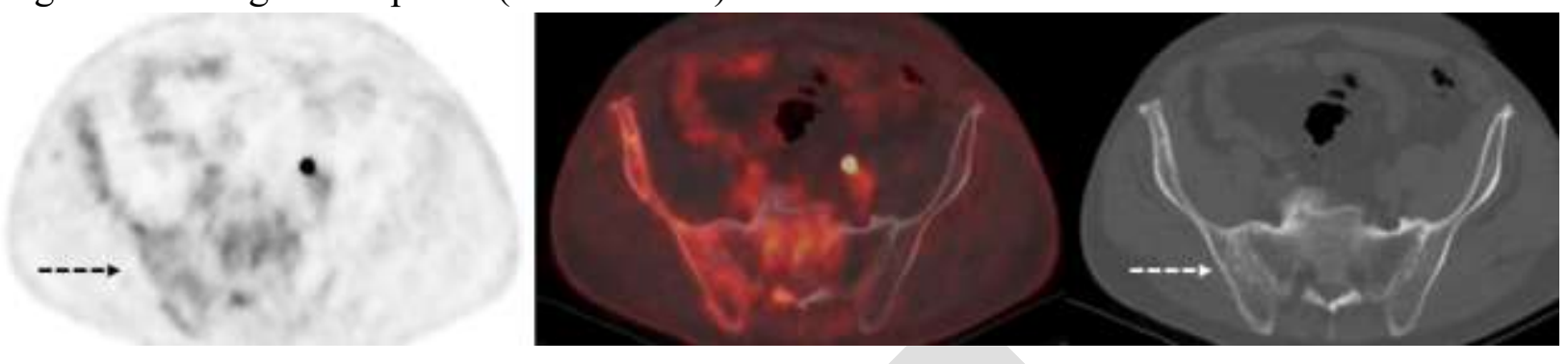

(B) In contrast, axial ${ }^{18} \mathrm{~F}$-DCFPyL PET, fused PET/CT and CT images show intense uptake at a site of prostate cancer metastasis in the inferior pubic ramus (solid arrow).
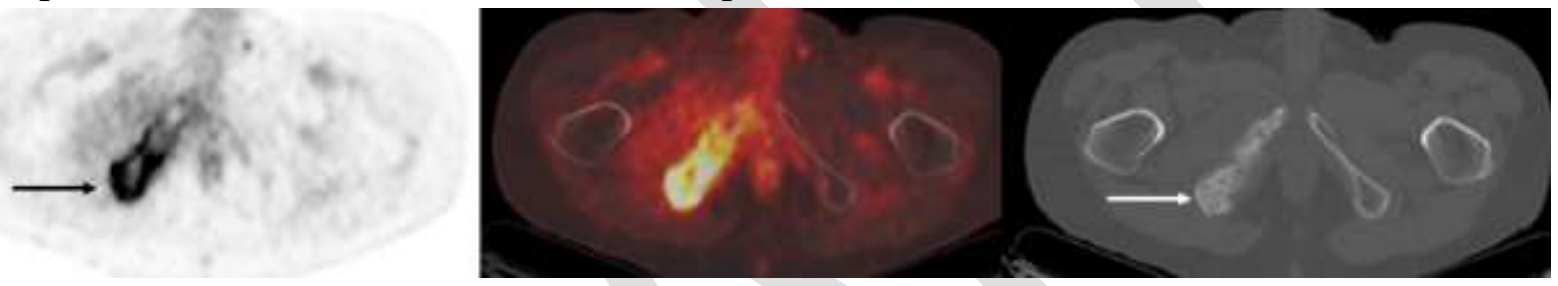
Fig. 11. A 69-year-old man with metastatic prostate cancer spread to retroperitoneal and cervical lymph nodes.

(A) Maximum intensity projection image shows intense ${ }^{18} \mathrm{~F}-\mathrm{DCFPyL}$ uptake in retroperitoneal (dotted arrows) and cervical nodes (solid arrows).
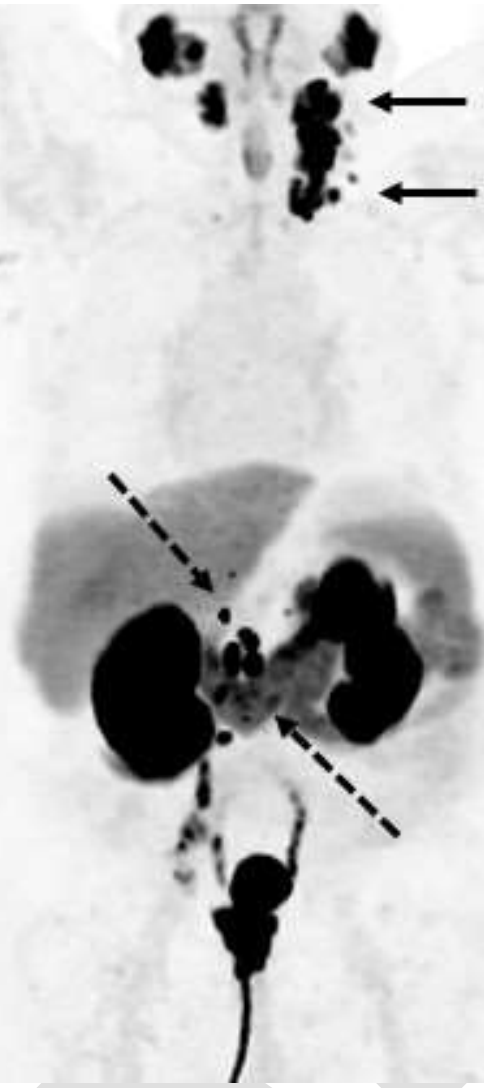

(B) Axial ${ }^{18}$ F-DCFPyL PET, fused PET/CT and CT images further delineate the cervical lymph node disease (arrow).
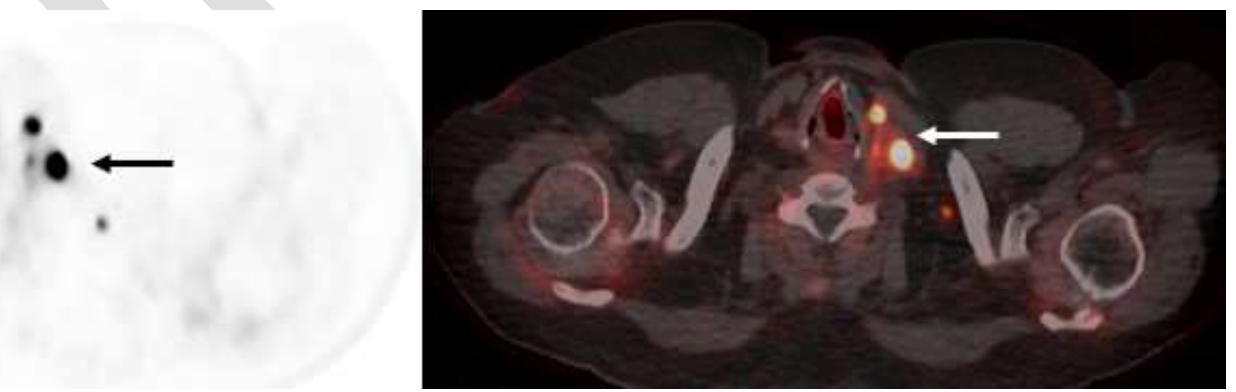
Fig. 12. A 62-year-old man 18 years post radical prostatectomy for pT2c N0 Gleason $7(3+4)$ prostate cancer and 10 years post salvage radiotherapy to prostate bed. Now rising PSA (latest $0.39 \mathrm{ng} / \mathrm{ml}$ ). Axial ${ }^{18} \mathrm{~F}$-DCFPyL PET, fused PET/MR images and contrast-enhanced Volumetric Interpolated Breath-hold (VIBE) MR image at the level of the prostate bed show a PSMA-avid nodule to the left of the surgical anastomosis (arrow) in keeping with local tumor recurrence (subsequently biopsy proven). No nodal or distant metastases were seen.

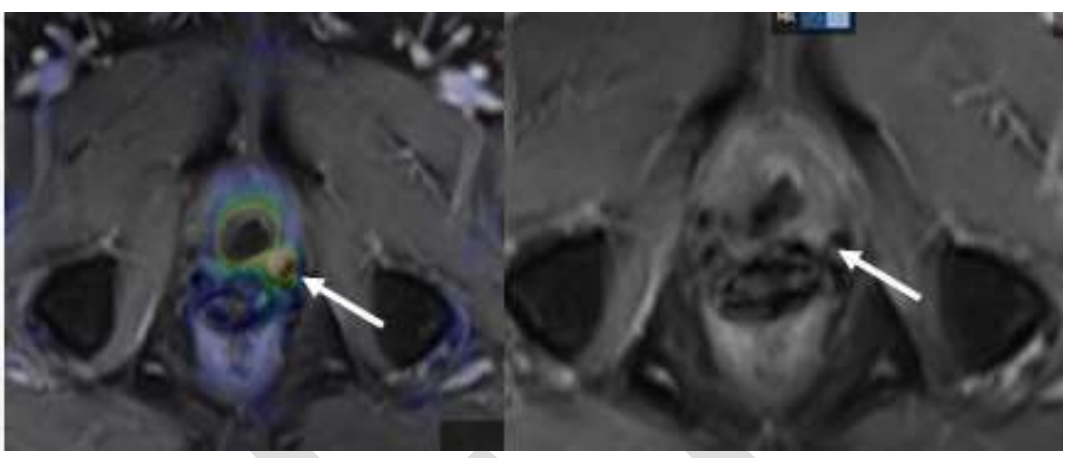


Fig. 13. A 70-year-old man 3 years post radical prostatectomy for pT3a, N0 Gleason 7 (4+3) prostate cancer, with negative surgical margins and biochemical recurrence (latest serum PSA $=$ $1.18 \mathrm{ng} / \mathrm{ml})$.

(A) Maximum intensity projection image shows multiple sites of abnormal ${ }^{18} \mathrm{~F}-\mathrm{DCFPyL}$ uptake.

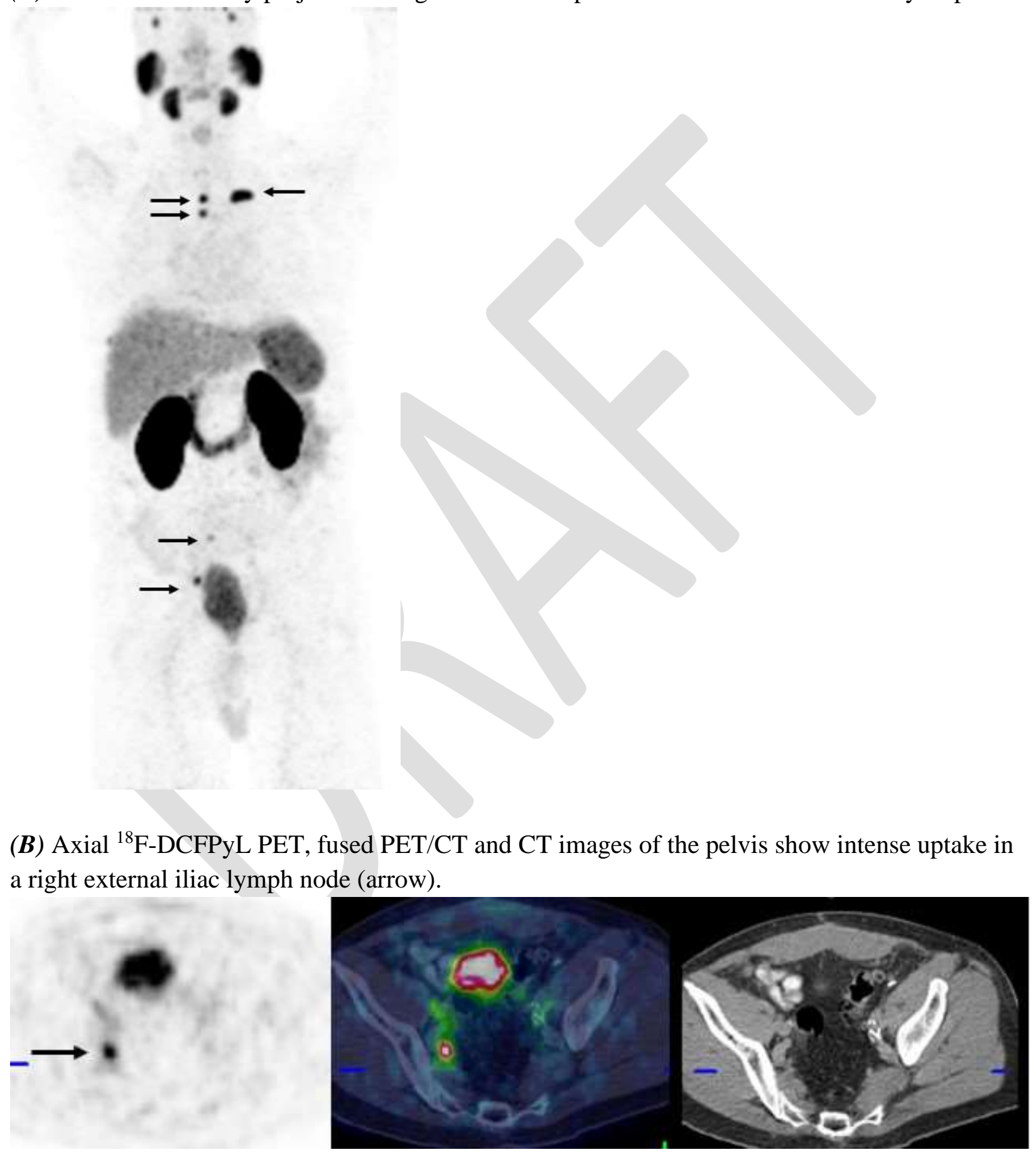

(B) Axial ${ }^{18}$ F-DCFPyL PET, fused PET/CT and CT images of the pelvis show intense uptake in a right external iliac lymph node (arrow). 
(C) Axial ${ }^{18}$ F-DCFPyL PET, fused PET/CT and CT images in the upper chest and sacrum show uptake in the skeleton in keeping with skeletal metastases [miM1b]. The patient was treated with androgen-deprivation therapy.

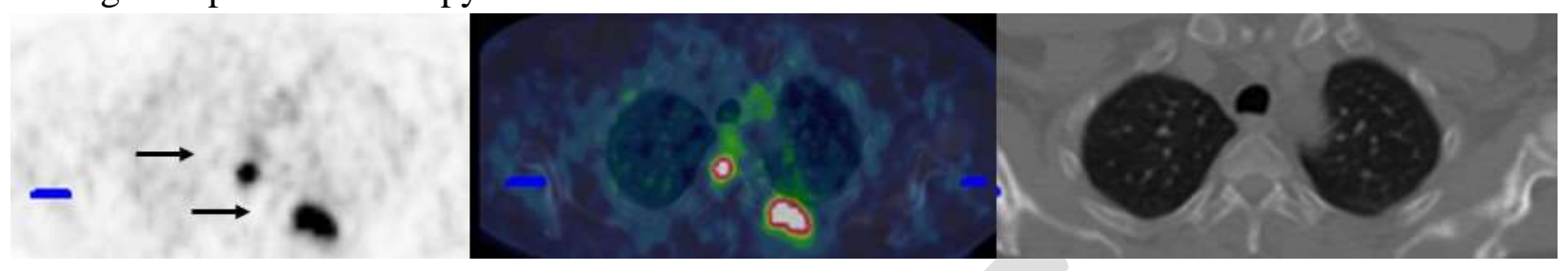

Fig. 14. A 67-year-old man 6 years post radical prostatectomy for pT3b N0, Gleason $7(4+3)$ prostate cancer and 4 years post salvage pelvic radiotherapy for biochemical recurrence with rising serum PSA (latest, $0.76 \mathrm{ng} / \mathrm{ml}$ ).

(A) Maximum intensity projection image shows a solitary site of intense ${ }^{18} \mathrm{~F}-\mathrm{DCFPyL}$ uptake in the right pelvis (arrow).

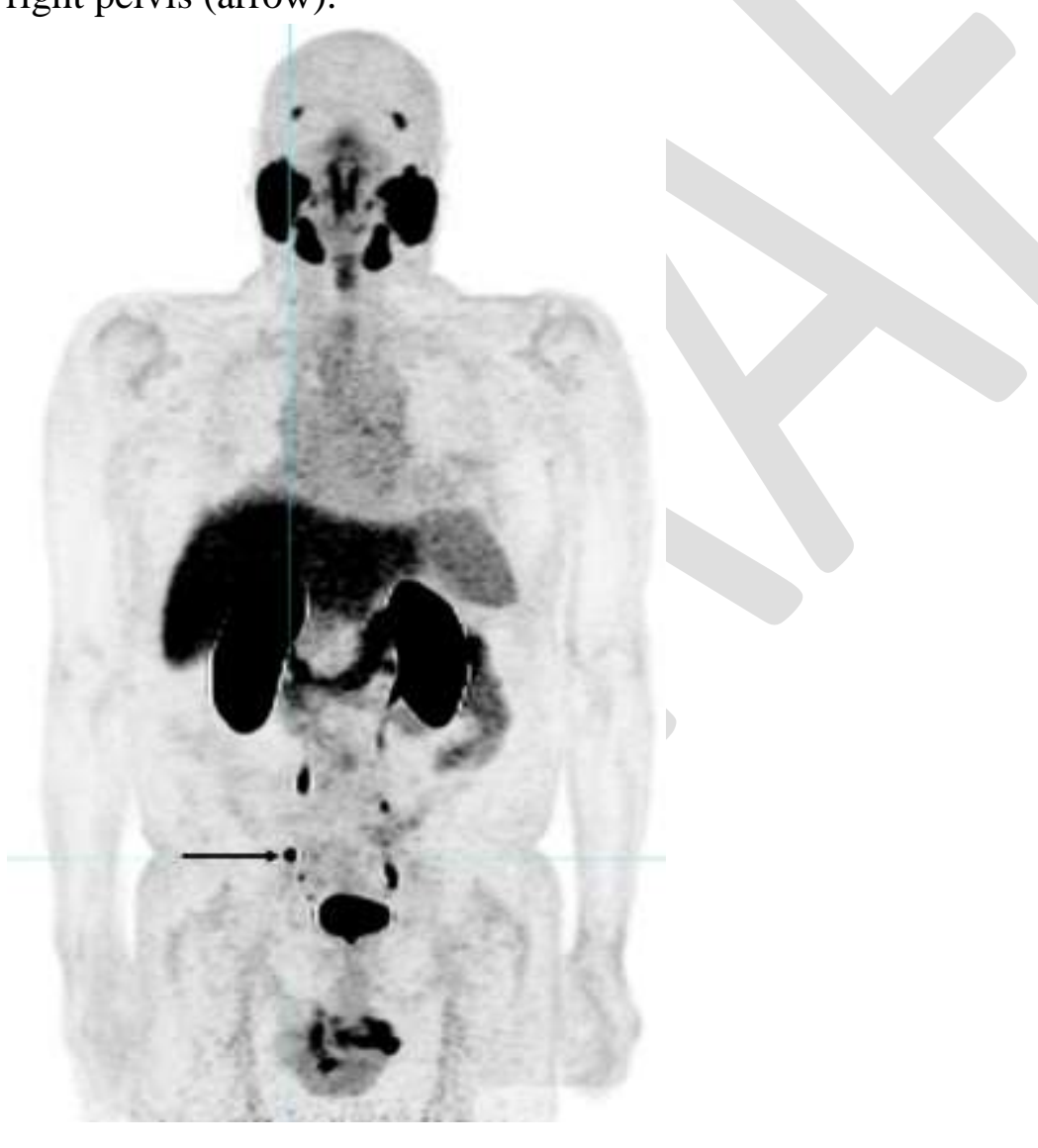


(B) Axial ${ }^{18}$ F-DCFPyL PET and fused PET/MR images show intense focal uptake in a right external iliac lymph node (crosshair). ${ }^{18} \mathrm{~F}$-DCFPyL PET/CT performed 4 months after salvage pelvic lymph node dissection with 1 of 11 resected pelvic nodes positive for metastasis showed no metastatic sites (not shown). Serum PSA was undetectable.

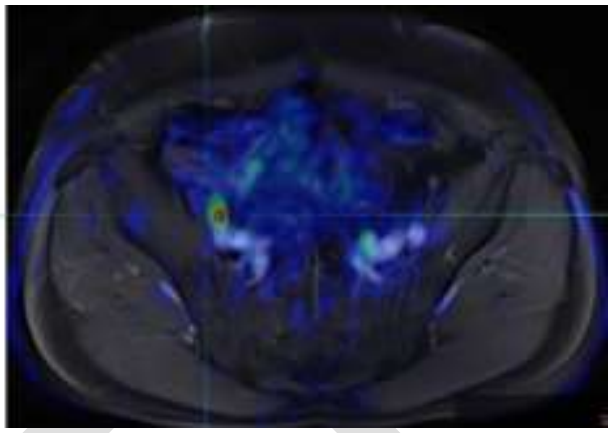

Fig. 15. A 63-year-old man with Gleason $7(4+3)$ prostate cancer initially treated with radical prostatectomy and progression of biochemical failure 8-months after pelvic radiotherapy for pelvic sidewall lymph node recurrence.

(A) Maximum intensity projection image shows normal ${ }^{18} \mathrm{~F}-\mathrm{DCFPyL}$ biodistribution with no evidence of disease recurrence.
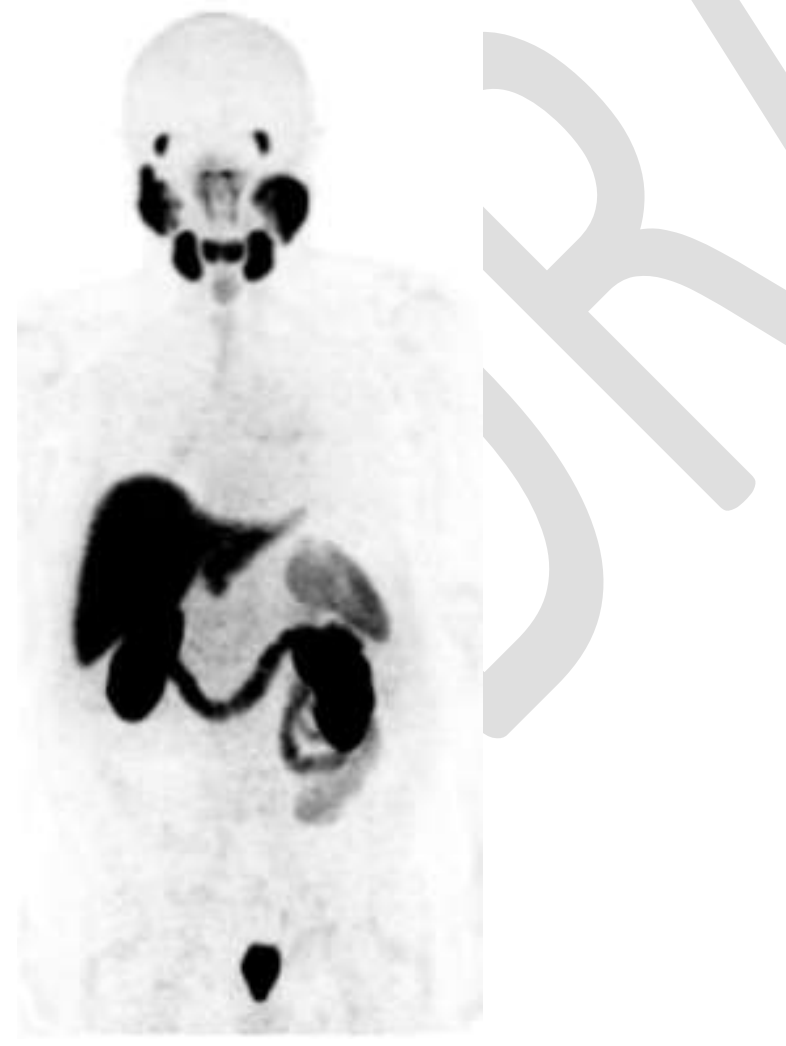
(B) Axial fused ${ }^{18} \mathrm{~F}$-DCFPyL PET/CT, CT, and PET images show a 2-cm segment 7 right hepatic lobe hypodensity (arrow), not seen previously, that was not PSMA-avid.
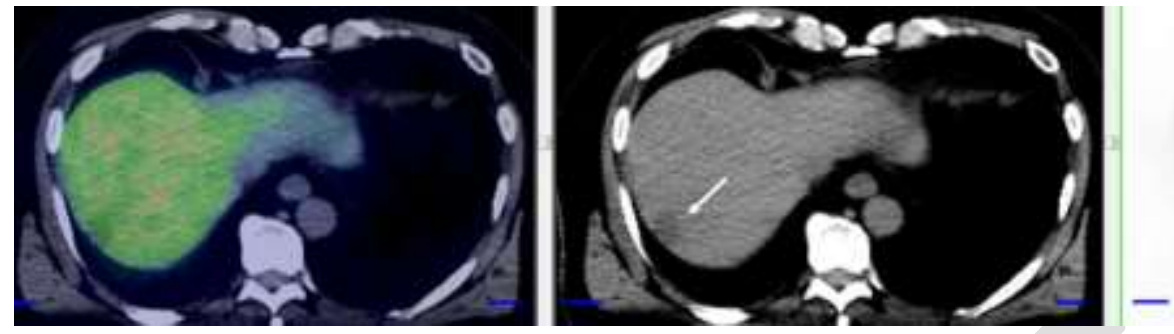

(C) Coronal contrast-enhanced CT images show multiple liver metastases (arrows), not previously seen. Biopsy of a segment 6 lesion confirmed adenocarcinoma with an immunohistochemistry profile consistent with prostatic origin.

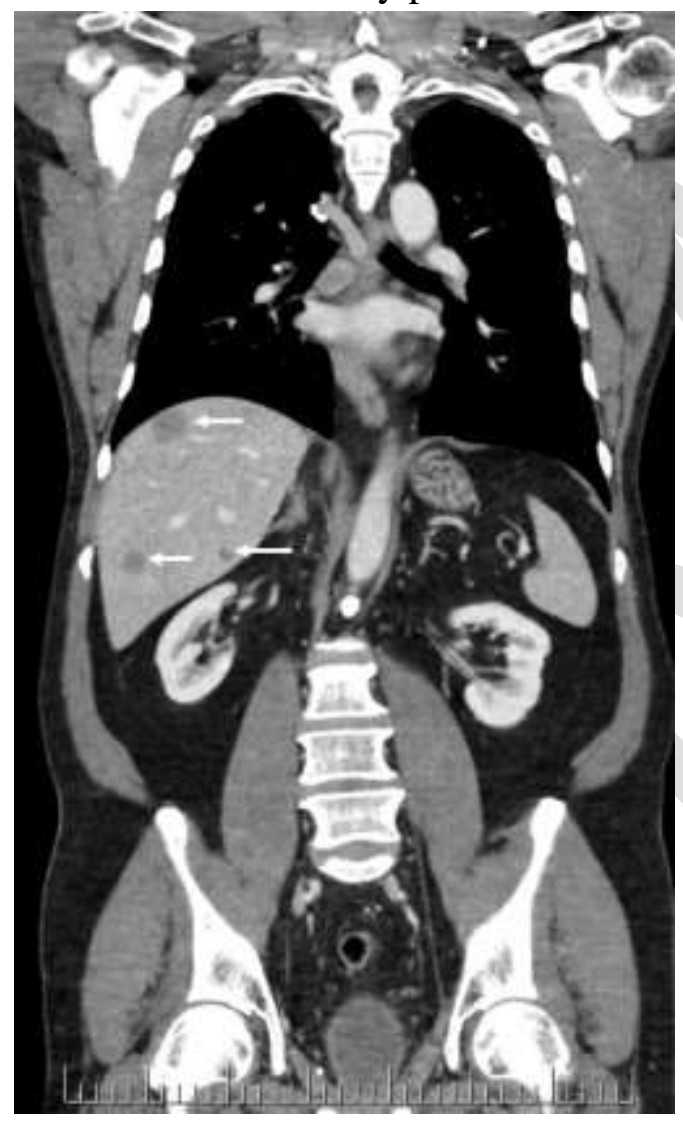


Fig. 16. A 58-year-old man with high-volume Gleason $9(4+5)$ prostate cancer with seminal vesicle invasion [cT3b]. Baseline serum PSA $=217 \mathrm{ng} / \mathrm{ml}$; negative conventional work-up. Maximum intensity ${ }^{18} \mathrm{~F}$-DCFPyL PET image and select coronal, axial and sagittal PET/MR images show extensive primary tumor (solid arrows), metastatic pelvic [miN] and common iliac lymph nodes [miM1a] (dotted arrows), as well as multiple bone metastases [miM1b] (arrowheads).

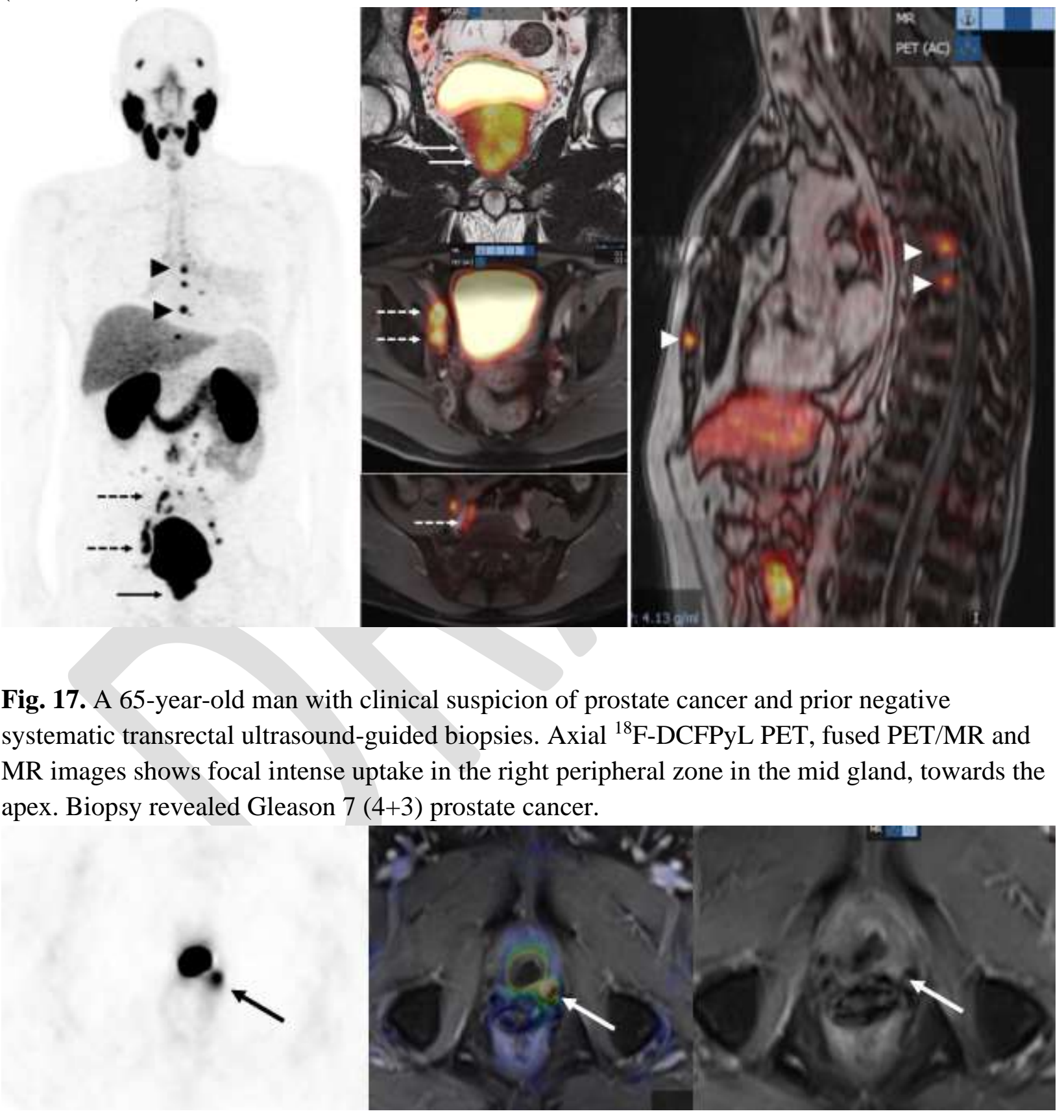




\begin{tabular}{|l|c|c|}
\hline \multicolumn{2}{|l|}{ Table 1. Suggested injected dose of common PSMA tracers and optimal uptake time } \\
\hline Tracer & Injected dose $*$ & Uptake time (min) \\
\hline${ }^{68} \mathrm{Ga}-\mathrm{PSMA}-11^{1}$ & $150 \pm 25 \mathrm{Mbq}$ & $60-90$ \\
\hline${ }^{18} \mathrm{~F}-\mathrm{DCFPyL}$ & $300 \pm 60 \mathrm{Mbq}$ & $60-120$ \\
\hline${ }^{18} \mathrm{~F}-\mathrm{PSMA}-1007^{3}$ & $300 \pm 60 \mathrm{Mbq}$ & $60-20$ \\
\hline
\end{tabular}

*Calculated for $75 \mathrm{~kg}$ man: $4 \pm 0.8 \mathrm{Mbq} / \mathrm{kg}$ for ${ }^{18} \mathrm{~F} \mathrm{~F}-\mathrm{DCFPyL}$ and ${ }^{18} \mathrm{~F}-\mathrm{PSMA}-1007$ and $2 \pm 0.33$ $\mathrm{Mbq} / \mathrm{kg}$ for ${ }^{68} \mathrm{Ga}-\mathrm{PSMA}-11$; and $5 \pm 1 \mathrm{Mbq} / \mathrm{kg}$ for ${ }^{18} \mathrm{~F}-\mathrm{FDG}$. These values can be adjusted according to body weight.

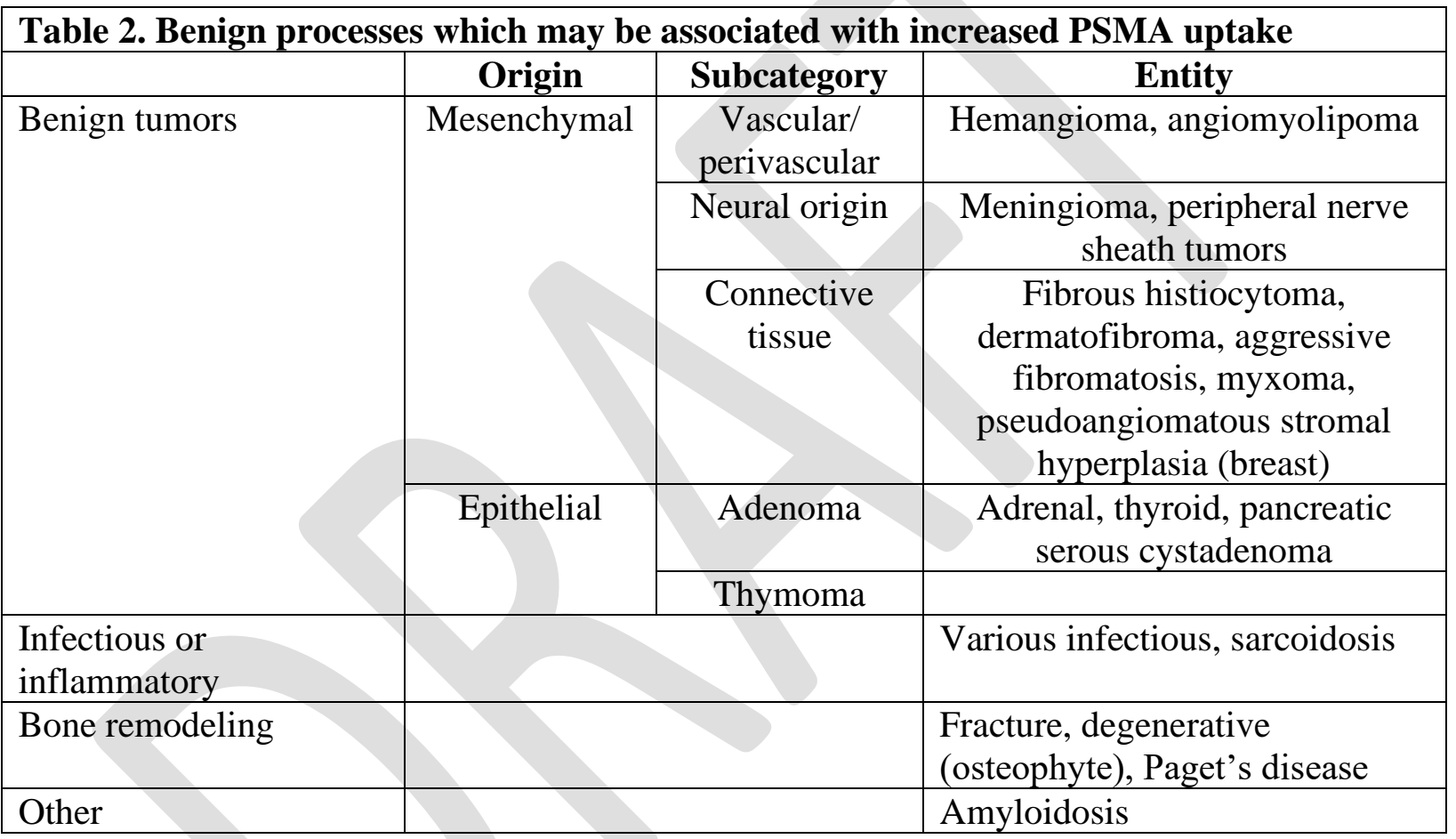




\begin{tabular}{|c|c|c|c|c|}
\hline & Indication & $\begin{array}{c}\text { Evidence } \\
\text { quality }\end{array}$ & $\begin{array}{l}\text { Recommendation } \\
\text { strength }\end{array}$ & Current recommendation \\
\hline Diagnosis & $\begin{array}{c}\text { Primary } \\
\text { diagnosis of } \\
\text { prostate cancer }\end{array}$ & 3 & 4 & $\begin{array}{l}\text { PSMA-targeted PET may be helpful } \\
\text { to identify clinically significant } \\
\text { prostate cancer when systematic } \\
\text { biopsies and MRI are negative }\end{array}$ \\
\hline $\begin{array}{l}\text { Primary } \\
\text { staging }\end{array}$ & $\begin{array}{l}\text { Primary staging } \\
\text { of high-risk } \\
\text { prostate cancer }\end{array}$ & 3 & 2 & $\begin{array}{l}\text { PSMA-targeted PET may be helpful } \\
\text { if a change in management is } \\
\text { contemplated }\end{array}$ \\
\hline \multirow[t]{3}{*}{$\begin{array}{l}\text { Castration- } \\
\text { sensitive }\end{array}$} & $\begin{array}{l}\text { Rising PSA after } \\
\text { prostatectomy; } \\
\text { locoregional } \\
\text { salvage } \\
\text { considered }\end{array}$ & 1 & 1 & $\begin{array}{l}\text { PSMA-targeted PET may be helpful } \\
\text { if locoregional salvage is considered }\end{array}$ \\
\hline & $\begin{array}{l}\text { Rising PSA after } \\
\text { radiotherapy; } \\
\text { locoregional } \\
\text { salvage } \\
\text { considered } \\
\end{array}$ & 2 & 2 & $\begin{array}{l}\text { PSMA-targeted PET may be helpful } \\
\text { if locoregional salvage is considered }\end{array}$ \\
\hline & $\begin{array}{l}\text { Potentially } \\
\text { oligometastatic, } \\
\text { castration- } \\
\text { sensitive } \\
\text { prostate cancer }\end{array}$ & 2 & 2 & $\begin{array}{l}\text { PSMA-targeted PET may be helpful } \\
\text { to confirm oligometastatic versus } \\
\text { extensive disease }\end{array}$ \\
\hline \multirow[t]{3}{*}{$\begin{array}{l}\text { Castration- } \\
\text { resistant }\end{array}$} & Non-metastatic & 3 & 2 & $\begin{array}{l}\text { PSMA-targeted PET may be helpful } \\
\text { if a change in management is } \\
\text { contemplated }\end{array}$ \\
\hline & $\begin{array}{l}\text { Metastatic with } \\
\text { PSA progression } \\
\text { (stable on CI*) }\end{array}$ & 4 & 3 & $\begin{array}{l}\text { PSMA-targeted PET should not be } \\
\text { routinely offered outside of a clinical } \\
\text { trial }\end{array}$ \\
\hline & $\begin{array}{l}\text { Metastatic with } \\
\text { radiographic } \\
\text { progression on } \\
\text { CI }\end{array}$ & 4 & 2 & $\begin{array}{l}\text { PSMA-targeted PET should not be } \\
\text { routinely offered outside of a clinical } \\
\text { trial }\end{array}$ \\
\hline
\end{tabular}

Quality of evidence: 1=high; 2=intermediate; 3=weak; 4=insufficient. Strength of evidence: $1=$ strong; $2=$ moderate; $3=$ weak. CI: conventional imaging (CT, bone scintigraphy). 